\title{
Análise da Relação entre Atributos de Automóveis e Valores Pessoais no Triângulo Mineiro
}

\author{
Analysis of the Relationship between Attributes of Automobiles and Personal \\ Values in the Triangulo Mineiro Region
}

\section{Tayanne Ferraz da Silva Poberschnigg' Márcio Lopes Pimenta ${ }^{2}$}

\section{Resumo}

O mercado consumidor de automóveis apresenta uma crescente expansão no Brasil. Esse crescimento traz consigo um aumento na concorrência, exigindo das empresas análises mais aprofundadas de modo a aprimorar as estratégias de marketing nesse setor. Nesse sentido, o objetivo deste trabalho é explicar o comportamento de escolha de veículos automotores na região do Triangulo Mineiro, estabelecendo a ligação entre atributos e valores pessoais, conforme a perspectiva da Teoria da Cadeia de Meios e Fins. Dessa forma, utilizou-se a técnica qualitativa Laddering para coleta de dados, análise e interpretação. Os principais atributos identificados foram Economia, Itens de Conforto, Marca e Preço. Estes atributos mostraram ligações com valores individuais relacionados a poder, realização e hedonismo. Estes valores, que influenciam o processo de decisão de compra, evidenciam que essas pessoas desejam ter sucesso profissional, bem-estar e felicidade.

Palavras-chave: Comportamento do Consumidor, Laddering, Veículos Automotores, Valores Pessoais.

\begin{abstract}
The automotive consumer market presents a growing expansion in Brazil. Such growing brings more competition to the sector, demanding deeper analyses in order to improve marketing strategies in this industry. Therefore, this paper aims to explain the choice behavior of automobiles in the Triângulo Mineiro's Region, establishing a link between attributes and personal values, according to the Means End Chain Theory. The Laddering qualitative technique was used to collect, analyze and interpret data. The main identified attributes were Economy, Comfort Items, Brand and Price. These attributes may promote linkages with individual values related to personal power, achievement and hedonism. These values, that influence the process of purchase decision, are an evidence that these people want to have professional success, wellbeing and happiness.
\end{abstract}

Keyword: Consumer Behavior, Laddering, Automotive Vehicles, Personal Values.

\footnotetext{
1 tayanne_92@hotmail.com, Brasil. Graduada em Administração pela Universidade Federal de Uberlândia - UFU. Av. João Naves de Ávila, 2121, Santa Mônica, CEP: 38400-902 - Uberlândia, MG - Brasil.

2 pimenta@fagen.ufu.br, Brasil. Professor da Universidade Federal de Uberlândia - UFU. Doutor em Engenharia de Produção pela Universidade Federal de São Carlos - UFSCAR. Av. João Naves de Ávila, 2121, Santa Mônica, CEP: 38400902 - Uberlândia, MG - Brasil.
}

Recebido em 26.08.2013

Aprovado em 06.05.2015

\section{Revista Administração em Diálogo}

ISSN 2178-0080 


\section{Introdução}

A relação entre valores e atributos é utilizada por alguns estudiosos de marketing que pretendem entender o comportamento do consumidor. Conforme Porto (2005), a percepção do significado do produto pode provocar reações emocionais nas pessoas. Para Cardoso e Kistmann (2008), os indivíduos tendem a escolher um produto com o qual se identificam, assim, muitas empresas procuram compreender os fatores motivacionais antes, durante e após o consumo, a fim de criar produtos que satisfaçam os indivíduos.

Partindo dessa ideia, autores como Fantini (2009), Oliveira, et al. (20IO) e Gonçalves Filho, Souki e Gonçalves (2009) abordam a conexão dos consumidores com a marca. Para esses autores, devido à concorrência no mercado, muitas organizações buscam consolidar suas marcas, atribuindo-lhes maior identidade, visto que a confiança na marca e o seu valor são alguns dos fatores que mais influenciam no processo de compra atualmente. Por isso, é importante identificar não somente quais atributos atraem os consumidores, mas também quais serão as consequências do consumo e quais valores dos indivíduos estão relacionados ao uso do produto.

O presente estudo tem como foco os consumidores de veículos automotores, um mercado que cresceu cerca de $145 \%$ entre 2002 e 2012 no Brasil e que, atualmente, representa I8,7\% do PIB industrial brasileiro (ANFAVEA, 20I4), sendo um importante setor para a economia do país. . Segundo Fernandes (2007), algumas montadoras não conseguem identificar as percepções dos consumidores, em razão da ênfase em conceitos operacionais. Esse fato pode prejudicar as organizações, considerando que os indivíduos percebem os carros como objetos de expressão de sua personalidade e de seus valores, como ressaltam Moyano e Lengler (20I3).

Assim, a compreensão da estrutura cognitiva de valores destes consumidores pode contribuir para entender o comportamento de compra destes produtos. Nesse sentido, o entendimento das conexões entre atributos, consequências e valores, envolvidos no processo de compra, pode permitir o direcionamento das ações de marketing a um posicionamento convergente com valores almejados pelos consumidores, desde a apresentação do produto até o estabelecimento de processos de comunicação (PIMENTA, et. al., 20o8). Poucos trabalhos estudados abordaram esse tema explicando 
as conexões entre atributos de veículos, consequências e valores (ESCUDERO; PRADO 2008; NUNES, et. al., 20I2).

De modo a explicar em profundidade a complexa relação entre as características dos veículos e valores pessoais, esse estudo é dirigido a responder a seguinte questão: Como se caracteriza a relação entre os atributos de veículos automotores com os valores pessoais e como a explicação dessa relação ajuda a entender o comportamento deste tipo de consumidor?

O objetivo geral deste trabalho é explicar o comportamento de consumidores na escolha de veículos automotores, estabelecendo a ligação entre atributos, consequências e valores pessoais conforme a perspectiva da Teoria da Cadeia de Meios e Fins. A seguir, é apresentado um referencial teórico expondo razões de compra de automóveis e sobre a influência de valores pessoais no consumo. O terceiro tópico explica o procedimento de coleta e a análise de dados das entrevistas em profundidade conduzidas com compradores de veículos na cidade de Uberlândia-MG. Na sequência são expostos os resultados e conclusões.

\section{Razões da Compra de Automóveis}

Diversos fatores guiam o comportamento de compra, variando desde motivações internas até influências externas. A soma desses fatores, cada qual com o grau de importância atribuído individualmente, consolida o processo de decisão de compra para cada consumidor (FANTINI, 2009). Foxall, et. al. (2006), destaca que experiências individuais de aprendizagem de compras passadas são relacionadas à forma de consumo, ao comportamento e às consequências percebidas. Nesse sentido, alguns autores destacam as marcas como importantes fatores influenciadores no processo de decisão de compra de automóveis (OLIVEIRA, et. al., 20IO; GONÇALVES FILHO; SOUKI; GONÇALVES, 2009; FANTINI, 2009). Para Oliveira, et. al. (2OIO), a marca é o principal vínculo de confiança entre o consumidor e a organização, uma vez que esta é a garantia de que o produto ou serviço trará os benefícios materiais e psicológicos almejados pelos compradores. Gonçalves Filho, Souki e Gonçalves (2009) destacam que as marcas podem influenciar consumidores a tomar decisões de compra baseadas em aspectos emocionais. 
Para Fantini (2009), com a intensificação da concorrência, as empresas vêm procurando configurar às suas marcas e aos seus produtos maior unicidade. Como consequência, proporcionam ao consumidor mais do que ele espera e/ou precisa, consolidando um maior caráter emocional ao momento da compra. Oliveira, et. al. (20IO), destaca que o ponto forte da marca emocional é justamente abranger não somente as necessidades, mas também os valores do seu público-alvo, estabelecendo uma conexão com este de forma singular. Por esta razão, devem ser trabalhados em conjunto fatores como: relacionamento, experiências sensoriais, imaginação e visão, a fim de conquistar a fidelidade e a confiança dos clientes. Fantini (2009) argumenta que as montadoras devem buscar formas de atuar com estes fatores humanos e comportamentais, no intuito de aprimorar estratégias de segmentação e diversificação de seus modelos.

Apesar disso, as classificações utilizadas pelas montadoras para seus modelos de veículos englobam conceitos operacionais, sendo, muitas vezes, incapazes de mensurar as percepções dos consumidores (FERNANDES, 2007). Isso acontece porque carros são produtos complexos, cujos atributos podem ser usados pelos consumidores para avaliação dos próprios produtos e formação de preferência de marca. Mas, para isso, é necessário que haja uma extensa busca de informações por parte dos compradores, como discorrem Chao e Gupta (I995).

De acordo com Mueller e Haan (2009), há fatores determinantes no momento da compra de automóveis, que englobam desde o desempenho do carro propriamente dito até sua classe, preço, tipo de combustível e grau de emissão de poluentes. Todos esses fatores combinados acabam tornando o processo decisório de aquisição de um novo veículo bastante heterogêneo e complexo. Pôde-se verificar também que o grau de pesquisa externa pré-compra é baixo, dado o elevado preço dos veículos novos. Tal comportamento pode ser explicado tanto pelo envolvimento de decisões heurísticas quanto pelo fato de a coleta de informações consistir em um processo contínuo, não limitado ao processo de compra. Ainda que, como ressalta Dias e Oliveira-Castro (2006), o objetivo fundamental do comportamento de busca de informações seria justamente atrelar ganhos em qualidade com diminuição dos custos. 
Para Fernandes (2007), as características situacionais e individuais também interferem na hora da compra de um veículo. Além destas, o autor destaca o fator econômico que também engloba as possibilidades de financiamento oferecidas. Estes elementos têm se fixado como um fator decisivo no momento de efetivar a compra para muitos consumidores. É o que indica a pesquisa realizada por Araújo, Araújo e Alexandre (2OII). Esses autores estudaram o processo de compra de automóveis, tendo como sujeitos de pesquisa estudantes universitários de uma instituição privada. Utilizando-se de análise fatorial, eles encontraram os seguintes fatores influenciadores no processo: importância da pesquisa em site, relação preço e marca, necessidade de financiamento na concessionária e necessidade de visita às lojas que vendem carros novos.

De acordo com Chao e Gupta (I995), o país de origem dos carros também é um fator que influencia o comprador no momento de tomar a decisão de compra do veículo automotivo. Grohmann, Battistella e Schoedler (20I2) argumentam que o modo mais clássico de se compreender o comportamento do consumidor envolve identificar quais atributos do produto são considerados significantes para a tomada de decisão dos indivíduos. Em sua pesquisa, os autores identificaram vários atributos, sendo que os mais relevantes foram resistência, dirigibilidade, atendimento pós-venda e itens de conforto. Já para Campos e Ramos (2002), que realizaram sua pesquisa com consumidores de carros que seguem a linha econômica, o que mais influencia a decisão de compra é o preço e a qualidade. Em acréscimo a essas variáveis, Sampaio, et. al., (2004), destaca como determinante para decisão de compra, a importância da aceitação social e da experiência, evidenciando a influência dos parentes e amigos na compra de automóveis; e Cerqueira, Silva e Farias (20I3), que realizaram seu estudo com consumidores de baixa renda, contribuem com a identificação da tecnologia e da satisfação pós-compra como fatores influenciadores.

Por fim, o trabalho de Lemos e Slongo (2OII) é especificamente baseado em veículos de alto valor. Esses autores destacam que automóveis são produtos complexos e suas características, avaliadas conforme os valores do consumidor, influenciam na decisão, sendo a preferência pelo produto formada através da análise do veículo e da marca. Contudo, mesmo a marca sendo importante, não é um atributo definitivo. O

\section{Revista Administração em Diálogo} ISSN 2178-0080 
mesmo ocorre com o preço e acessórios. Para consumidores desse tipo de veículo, as variáveis que mais importam são: design, segurança, beleza e atendimento que lhes é dado na compra. Estas contribuições reforçam a importância de que os profissionais envolvidos na fabricação e venda de automóveis conheçam o perfil dos clientes e seus valores.

Conforme o conteúdo deste subtópico percebe-se que existem diversos tipos de fatores influenciadores de compra de automóveis. O próximo subtópico trará especificamente da influência de valores pessoais no comportamento de compra.

\section{Influência de Valores Pessoais no Consumo de Automóveis}

Em razão das transformações socioeconômicas do século XX, intensificou-se a análise das organizações por meio de seus aspectos intangíveis, como sua capacidade de gerar valor por meio do conhecimento de fatores como o comportamento do consumidor (Sampaio, et. al., 2004). Para esses autores, as pessoas não buscam somente bens ou serviços, mas benefícios que serão adquiridos com o consumo dos mesmos. Torna-se, então, necessário entender a relação entre os valores pessoais e a forma como os indivíduos valorizam os produtos ou serviços a fim de compreender seu comportamento.

Porto (2005) acredita que a estratégia de marketing baseada em valores pessoais tende a ser mais persuasiva do que a baseada em experiências de compra. Esse autor afirma que os valores pessoais correspondem aos objetivos de vida dos indivíduos e que o significado do produto é representado pela reação de afeto que o mesmo desperta no consumidor. Segundo Muralie e Mittal (2OII), os valores considerados em uma situação de compra são resultados da emoção da experiência de consumo. Seguindo esse raciocínio, Ramalho e Ayrosa (20o9) destacam que os carros incorporam cada vez mais valores pessoais e se transformam em objetos de desejo, adorno e identificação de grupos. No momento em que o indivíduo se identifica com o bem, ele reconhece suas características pessoais neste ou características que almeja, estabelecendo a extensão de si no objeto.

Valores representam as crenças e os comportamentos aceitáveis por parte das pessoas. De acordo com Blackwell, Miniard e Engel (2005), valores são elementos importantes para a compreensão e previsão do comportamento do consumidor, 
diferenciando-se das atitudes pela sua ligação a questões mais duradouras e intrínsecas. Para esses autores, a compreensão da estrutura de valores pode ser utilizada para identificar as razões que levam as pessoas a agirem de determinada maneira, pois representam crenças sobre determinados estados a serem alcançados. Para Baker, Thompson e Engelken (2004), os valores contribuem para o entendimento psicológico do indivíduo, no sentido de descobrir quais são os critérios que o levam a selecionar e julgar pessoas e eventos, inclusive as situações de decisão de compra.

Diversos autores estudam a influência de valores pessoais no comportamento do consumidor. Estes citam como relevante, ao longo da literatura deste assunto, os modelos da escala de valores de Rokeach e da escala de valores de Schwartz (GUTMAN, I982; GUTMAN, I99I; NASPETTI; ZANOLI, 2004; BLACKWELL; MINIARD; ENGEL, 2005; PIMENTA, et. al., 20I2).

Segundo Rokeach (1973), valores consistem em estruturas psicológicas resultantes de experiências pessoais, sociais e culturais. Este autor reduziu, através de entrevistas, um vasto número de valores mencionados na literatura. Baseando-se nas análises empíricas de sua pesquisa, o autor concluiu que existem dois tipos básicos de valores: terminais (representando metas de vida desejadas / estados finais desejáveis); e instrumentais (representando modos de viver desejados / modos de condutas). Em complemento a esta abordagem, Schwartz (1992), mostrou que valores podem ser categorizados dentro de dez domínios, simplificados em quatro domínios maiores, conforme mostra o Quadro I a seguir. 
Quadro I - Modelo de Domínios de Valor do Schwartz

\begin{tabular}{|c|c|}
\hline Dimensões de Valor & Tipos de Valor \\
\hline \multirow{3}{*}{ Autoaprimoramento } & Poder: poder social, preservação da imagem pública, controle/autoridade, riqueza \\
\hline & Realização: sucesso, capacidade, ambição, diversão \\
\hline & Hedonismo: prazer, aproveitar a vida, valores individuais \\
\hline \multirow{2}{*}{ Abertura à Mudança } & Estimulação: vida sem rotina, excitante e desafiante \\
\hline & Autodirecionamento: criatividade, curiosidade, liberdade \\
\hline \multirow[t]{2}{*}{ Autotranscedência } & Universalismo: cabeça aberta, justiça social, igualdade, proteção para o ambiente \\
\hline & Benevolência: visar ao bem-estar das pessoas, solicitude, honestidade, clemência \\
\hline \multirow{3}{*}{ Conservadorismo } & Tradição: compromisso, respeito e aceitação da cultura a qual está inserido, humildade, gratidão \\
\hline & $\begin{array}{l}\text { Conformidade: disciplina, honradez, obediência às normas e restrição de ações que possam prejudicar } \\
\text { os outros }\end{array}$ \\
\hline & Segurança: ordem, harmonia e segurança social e individual, limpeza, saúde \\
\hline
\end{tabular}

Fonte: Adaptado de Schwartz (1992).

Destacados esses aspectos, cabe destacar quais valores pessoais estariam envolvidos com a aquisição de um automóvel. Carvalheiro (2OI2) e Porto e Torres (2OI4) apontam que o setor automobilístico apresenta várias alternativas de marcas e subtipos, como sedan luxuoso, utilitário esportivo e compacto luxuoso, sendo que a escolha por determinado modelo vai depender do objetivo do consumidor ao adquirir o automóvel.

Conforme Porto e Torres (2OI4), os valores pessoais influenciam a importância atribuída aos critérios de decisão, levando-se em consideração tanto a escolha da marca quanto do tipo de veículo. Para esses autores, os valores autodeterminação, benevolência e realização impactam positivamente na preferência por marcas e sedans percebidos como luxuosos. Para carros compactos luxuosos, o valor que sobressaiu foi a estimulação e para carros utilitários esportivos os valores foram poder, autodeterminação, benevolência, realização e segurança.

Fernandes (2007) destacou a influência social, a propensão à economia, a percepção de valor e, por último, o consumo consciente. Além disso, o autor evidenciou a relação entre a preferência de compra e diversos valores: status, desenvolvimento intelectual, valorização da família, otimismo, liderança, conservadorismo, grau de imediatismo e profissionalismo, em ordem de importância.

\section{Revista Administração em Diálogo} ISSN 2178-0080 
Partindo para uma análise mais aprofundada, outros estudiosos buscaram relacionar o perfil do consumidor com a escolha automóvel. Escudero e Prado (2008) realizaram uma pesquisa com consumidores de automóveis esportivos compactos abrangendo dois segmentos: pessoas da classe A e classe B no Brasil. A classe A foi denominada como o grupo "Segurança e Bem-estar", em que os indivíduos correspondentes a ela buscam respeito próprio, senso de realização, prosperidade, sabedoria, entre outros. A classe B foi denominada como o grupo "Individualismo e Hedonismo" que busca prazer, excitação, reconhecimento social, individualidade, jovialidade, o belo e o moderno. Assim, um indivíduo que visa um carro bonito tem por objetivo maior chamar a atenção de terceiros, e isso leva a crer que o mesmo possa valorizar o reconhecimento social ou sua individualidade. O design seria a característica que esse consumidor mais prezaria na compra.

Nunes, et. al,.(20I2) realizaram uma pesquisa no Ceará envolvendo jovens, a fim de relacionar os atributos de veículos automotores com os valores pessoais. Ao todo foram encontrados 9 valores, sendo eles: desenvolvimento do país, preocupação com o futuro, privacidade, qualidade de vida, satisfação pessoal, segurança, sentir-se bem, status e vida saudável. Já Carvalheiro (20I2) concluiu em seus estudos sobre o comprometimento afetivo dos consumidores com automóveis de luxo, que a qualidade e a satisfação exercem grande impacto neste sentido.

Vinson, Scott e Lamont (1977) concluíram que indivíduos mais liberais têm valores como: respeito próprio, perdão, qualidade de vida e busca por uma vida excitante. Dessa forma, esses consumidores procuram produtos duráveis, com baixa emissão de poluentes e potentes. Além disso, demonstram admiração por carros compactos. Já os consumidores mais tradicionais têm valores como a segurança nacional, a educação, o reconhecimento social e o pronto atendimento em queixas. Esses consumidores buscam produtos que proporcionem prestígio e que tenham o interior espaçoso e luxuoso.

Como pode ser percebido, ao adquirir um bem, os indivíduos procuram se identificar com o mesmo, o que ressalta a relevância dos valores pessoais no estudo do comportamento do consumidor. Há muitos valores ligados à compra de veículos automotores, de modo que os resultados dessa pesquisa podem reforçar o que foi 
apresentado nessa revisão bibliográfica e/ou acrescentar novas informações para futuras investigações.

\section{Metodologia}

Conforme a perspectiva de Andrade (I999), essa pesquisa se caracteriza com o qualitativa e explicativa, por estar voltada à identificação de elementos subjetivos e à análise destas variáveis a fim de mostrar suas inter-relações e efeitos. Para tanto, primeiramente foi realizada uma pesquisa bibliográfica, consultando-se pesquisas relacionadas a influência de valores no consumo por meio de livros, artigos, dissertações e documentos setoriais. Em sequência, realizou-se um estudo de campo, de modo a aprofundar uma realidade específica por meio de entrevistas, as quais têm a finalidade de captar explicações e interpretações dessa realidade (GIL, 2002).

Foram entrevistados vinte e seis consumidores em uma concessionária em Uberlândia-MG. As entrevistas foram realizadas no período de novembro de 201 a janeiro de 20I2. É importante ressaltar que os entrevistados foram conduzidos a uma sala com assentos, onde não havia interferência dos funcionários e nem ruídos que comprometessem o áudio da gravação. Os consumidores foram abordados durante sua espera pelo atendimento das primeiras revisões ou retirada do veículo recém-comprado, sendo alertados que a identidade destes não seria revelada.

Antes de iniciar a entrevista, foi aplicado um questionário sociodemográfico para caracterização do perfil dos entrevistados. Em média, essas pessoas tinham 43 anos, com uma renda familiar aproximada de $\mathrm{R} \$ 4.5 \mathrm{Oo}, \mathrm{oO}$, com 3 moradores por domicílio. Em relação ao tempo de compra, Io clientes $(38,46 \%)$ adquiriram seu veículo no período de I até 3 meses, 5 ( $19,24 \%)$ no período de 3 até 5 meses, 4 ( $5,4 \%)$ no período de 5 até 8 meses e, por fim, 7 clientes $(26,9 \%)$ realizaram a compra entre 8 e ı 2 meses.

A técnica Laddering orientou a coleta, análise e interpretação dos dados, contribuído para encontrar ligações entre atributos percebidos, consequências e valores, seguindo, assim, o modelo de cadeias meios-fim (REYNOLDS; GUTMAN, 1988). A análise dos dados coletados nas entrevistas é foi a partir do resumo dos elementos-chave (atributos-consequências-valores), da representação da conexão entre esses elementos e, posteriormente, da construção de um mapa hierárquico de valor que faz associações 
sobre os níveis de abstração. A interpretação do resultado permite ao pesquisador compreender a motivação do consumidor para uma determinada classe de produtos ou serviços.

$\mathrm{Na}$ fase da coleta de dados, inicialmente é pedido que o entrevistado aborde os atributos do produto, o que pode ser feito de três maneiras: I) escolha de três (triadic sorting), em que se pede para o entrevistado distinguir três marcas diferentes de um produto; 2) diferenças de preferência de consumo, envolvendo a preferência de uma marca em comparação às demais; e 3) diferenças de ocasião, onde se deve sugerir um contexto de consumo ao entrevistado (REYNOLDS; GUTMAN, I988). Embora não houvesse roteiro de entrevista, a pergunta inicial feita aos entrevistados neste estudo era voltada a identificar quais aspectos, características ou atributos a pessoa levou em consideração na escolha do modelo do carro e sua marca.

Para Veludo-de-Oliveira e Ikeda (2004) o entrevistador deve motivar o entrevistado a responder com perguntas repetitivas, para que se possa chegar às consequências e aos valores. Contudo, recomenda-se alertar ao respondente as características da técnica utilizada, pois ele pode acreditar que as questões são muito evidentes e cansativas. Assim, foi questionado aos consumidores perguntas como "por que esse atributo é importante?”, "qual é a sensação de ter um carro com (ou sem) isso?”, “o que isso significa pra você?”, para identificar os elementos e seus significados.

Durante essa fase ocorreram problemas como o entrevistado não saber responder ou, então, ficar intimidado com a pergunta, por ser algo considerado muito pessoal. Diante desses obstáculos, foram utilizadas algumas técnicas propostas por Reynolds e Gutman (I988) para auxiliar na solução desses problemas. Podem ser citadas: evocar um contexto situacional, postular a falta de um objeto ou sentimento, laddering negativo, contraste com a regressão da idade, abordagem de terceira pessoa e técnicas de redirecionamento (silêncio e checagem da comunicação). Além disso, em determinadas entrevistas, parou-se de abordar um assunto quando não se conseguiu avançar na escala, retornando a ela posteriormente durante a entrevista, a fim de desinibir os entrevistados.

A fase da análise dos dados, por sua vez, abrange quatro etapas: a análise de conteúdo, o desenvolvimento da matriz implicação, a construção do mapa hierárquico de valor e a determinação das orientações de percepção dominante:

\section{Revista Administração em Diálogo} ISSN 2178-0080 
I) Na primeira etapa as entrevistas gravadas foram transcritas, lendo-se o texto e separando as orações, a fim de classificar os elementos em atributos, consequências ou valores pessoais; esses elementos foram codificados - atribuição de números - para, então, interpretar-se o referencial de codificação - conjunto de códigos. Assim, a análise de conteúdo torna os textos menos complexos ao sintetizar suas características em uma descrição objetiva, cabendo aos pesquisadores conciliarem fidedignidade e validade no processo (VELUDO-DE-OLIVEIRA; IKEDA, 2004; BAUER; GASKELL, 2003).

2) Conforme Veludo-de-Oliveira e Ikeda (2004) e Reynolds e Gutman (I988), na segunda etapa forma-se a matriz para verificar quantas vezes determinado elemento é direcionado a outro, o que é feito a partir da análise das ladders, a relação entre os elementos pode ser tanto direta quanto indireta.

3) A terceira etapa consiste em mapear as relações relevantes entre os elementos. Cria-se então o mapa hierárquico de valor, por meio das relações numéricas dispostas na matriz de implicação.

4) Na quarta etapa devem ser observadas quais são as principais cadeias, que são aquelas que possuem maior soma de relações entre os elementos.

A matriz implicação e o mapa hierárquico de valor foram obtidos através do programa MECanalyst Plus. O ponto de corte escolhido para elaborar o mapa hierárquico de valor foi 3 , o que indica que as cadeias no mapa deste estudo apresentam 3 ou mais relações diretas ou indiretas entre os elementos - atributos, consequências e valores. Escolheu-se este ponto de corte por abranger aproximadamente 8o\% das ligações totais da matriz de implicação, conforme sugerido por Reynolds e Gutman (I988). Dessa forma, são incluídos no mapa somente os elementos que foram mais citados pelos entrevistados e que mais estabeleceram conexões entre si, a fim de que os resultados fossem mais consistentes para explicar o fenômeno estudado.

\section{Resultados}

Este tópico apresenta três tipos de conteúdo presentes na análise realizada: I) a explicação dos atributos, consequências e valores encontrados por meio da análise de conteúdo das entrevistas (Quadro 2); 2) As conexões entre esses elementos obtidas em 
uma matriz numérica (Tabela I); 3) a análise de um mapa cognitivo que representa os comportamentos predominantes dos entrevistados na escolha de veículos (Figura 2).

Por meio da análise de conteúdo das entrevistas realizadas, foram identificados 37 elementos, sendo I3 atributos, I5 consequências e 9 valores. O Quadro 2 mostra de que forma esses elementos foram agrupados, como também seus respectivos códigos:

Quadro 2 - Tabela de Elementos: Atributos, Consequências e Valores

\begin{tabular}{rlrlll}
\hline & Atributos & & Consequências & & Valores \\
\hline 1 & Acabamento Interno & 14 & Ajudar as Pessoas & 29 & Altruísmo \\
2 & Aparelho de Som & 15 & Atendimento Diferenciado & 30 & Autoestima/Ego \\
3 & Bluetooth & 16 & Conforto & 31 & Bem-estar pessoal e da família \\
4 & Suspensão Robusta & 17 & Desempenho Profissional & 32 & Felicidade \\
5 & Desenho/Aparência & 18 & Evitar Transtornos & 33 & Gratidão \\
6 & Econômico & 19 & Economizar Tempo & 34 & Realização \\
7 & Espaço Interno & 20 & Honrar Compromissos & 35 & Respeito \\
8 & Itens de Conforto & 21 & Lazer & 36 & Segurança/Confiança \\
9 & Itens de Segurança & 22 & Levar mais pessoas/Amigos & 37 & Tranquilidade \\
10 & Marca & 23 & Menor manutenção/Prevenir estrago & & \\
11 & Motorização & 24 & Mostrar Simplicidade & & \\
12 & Preço & 25 & Prazer de Dirigir & & \\
13 & Resistência & 26 & Sobrar mais dinheiro & & \\
& & 27 & Tempo de Garantia & & \\
\hline
\end{tabular}

Fonte: Dados da pesquisa.

Em relação ao significado dos atributos, o "Acabamento Interno" refere-se ao visual interno do automóvel e a não ouvir barulho de peças do carro enquanto se dirigi; "Aparelho de Som" e "Bluetooth" são o próprio item em si. Já "Suspensão Robusta” está ligada a carros fora de estrada, muito utilizado por pessoas que frequentam fazendas e necessitam de um carro maior e mais alto, que tem firmeza para dirigir; o "Desenho/Aparência" envolve a cor do carro, seu design com "traseira e frente bonitas" nas palavras dos entrevistados.

O atributo "Econômico" envolve o consumo de combustível e o baixo custo com manutenção e/ou peças mais baratas; "Espaço Interno" relaciona-se ao tamanho do porta-malas (grande) e ao motorista e os passageiros não ficarem desconfortáveis no carro devido ao pequeno espaço; os "Itens de Conforto" são direção hidráulica, vidro

\section{Revista Administração em Diálogo} ISSN 2178-0080 
elétrico, ar condicionado e bancos macios; e os "Itens de Segurança” citados são airbag'e freio ABS.

A "Marca" é pelo fato da Chevrolet ser uma marca conhecida e de confiança no mercado e a "Motorização" envolve a potência do motor, a capacidade de aceleração. O atributo "Preço" relaciona-se à compatibilidade com o salário, ao custo-benefício de ter um carro completo e não tão caro em relação às demais agências e às regalias, as quais envolvem desconto, imposto pago e garantia. Quanto à "Resistência", a mesma está ligada durabilidade, a estabilidade e a robustez do carro.

Em relação às consequências, "Ajudar as Pessoas” envolve transportar pessoas com conforto, fazendo com que se sintam bem, e também proporcionar condições melhores para os membros familiares; "Atendimento Diferenciado" relaciona-se tanto à receptividade na concessionária, importando aqui a demonstração de atenção e preocupação com os clientes, quanto à facilidade de fazer manutenção e de encontrar peças para reposição.

O "Conforto" está ligado ao modo como a pessoa está acomodada no carro (evitando o cansaço), à conveniência e à comodidade (rápida mobilidade), à facilidade de direção, à tecnologia e ao espaço interno do carro. O “Desempenho Profissional” referese a trabalhar mais e melhor, cumprindo os compromissos e investindo no negócio. "Evitar Transtornos" é evitar ter problemas mecânicos com o carro e/ou sentir medo devido à falta de segurança do veículo, envolvendo a estabilidade e a confiança que se tem no carro; e, em outros casos, evitar estresse ligado à inadimplência durante o financiamento ao comprar um carro de acordo com a renda, por exemplo, a fim de preservar a vida.

"Economizar Tempo" está relacionado à agilidade que o carro proporciona, evitando que o cliente perca tempo ao chegar mais rápido nos lugares que deseja ir. Já “Honrar Compromissos” refere-se a ter responsabilidade e cumprir seus compromissos, seja em questões de pontualidade ou em questões de pagamentos de dívidas. O “Lazer” é ter dinheiro para poder investir em viagens, gastos básicos e manutenção da casa e do carro; "Levar mais pessoas/Amigos" é ter um carro com mais espaços para passear com a família e os amigos. 
A consequência "Menor manutenção/Prevenir estrago" está ligada tanto a manutenção preventiva para evitar gastos com estrago, quanto com o carro ter qualidade, durabilidade e peças mais baratas. "Mostrar Simplicidade", por sua vez, é o consumidor utilizar o carro sem a finalidade de esnobá-lo perante aos demais; "Prazer de Dirigir" envolve a sensação de dirigir que pode estar conectada a condução ou a aparência do carro.

"Sobrar mais dinheiro" é ter um carro que evita gastos, a fim de formar poupança e/ou economizar para ajudar no orçamento familiar; "Tempo de Garantia” relaciona-se à garantia do carro, a qual os entrevistados consideraram relevante; e "Tradição" é a confiança que se tem em comprar um carro de uma marca que está há muito tempo no mercado e que é recomendada por familiares e amigos.

Enfim, têm-se os valores. O "Altruísmo" significa fazer algo em prol do próximo; "Autoestima/Ego" tem relação com a projeção da aparência do carro à aparência da pessoa, como também com o fato do indivíduo se sentir importante e estar bem consigo. O valor "Bem-estar pessoal e da família" envolve se sentir bem ao dirigir e ao honrar um compromisso, proporcionar conforto para a família e ter qualidade de vida, como mencionou um entrevistado: “Quem trabalha muito, como é o meu caso, no mínimo tem que morar bem, comer bem, vestir bem, ouvir uma boa música”.

A "Felicidade" está ligada a ter dinheiro, segurança e saúde e a ausência de estresse e problemas; "Gratidão" é um valor religioso, espiritual, e "Realização" relaciona-se a ter a sensação de dever cumprido ao alcançar um objetivo e/ou adquirir um bem material através do seu esforço. "Respeito" envolve a reciprocidade e a responsabilidade com os demais indivíduos e "Segurança/Confiança", a dirigir educadamente e ter um carro seguro; por fim, "Tranquilidade" é um valor que se refere às sensações de alívio, calma e paz.

Este tópico mostrou os significados destes elementos oriundos da percepção dos consumidores. Além desta análise inicial, e também com base nas entrevistas, pôde-se formar as conexões entre atributos, consequências e valores. Para tanto, foi elaborada a matriz implicação, representada pela Tabela I. Essa matriz apresenta relações sobre de quantas vezes cada atributo foi ligado diretamente (lado esquerdo da vírgula) ou 
indiretamente (lado direito da vírgula) a uma consequência, ocorrendo o mesmo com a ligação entre consequências e valores.

\section{Matriz de Implicação}

A matriz implicação fornece, como sugerem Reynolds e Gutman (I988), quantas vezes cada elemento está ligado a outro e é útil para verificar quais são as conexões predominantes entre atributos, consequências e valores, sendo a base para a construção do mapa hierárquico. A matriz apresenta dois tipos de relação: direta, a relação de um elemento com o do nível seguinte; e indireta, a relação de um elemento com outro de qualquer nível acima, que não seja o seguinte.

Por exemplo, o atributo "Desenho/Aparência" obteve 5 ligações diretas com a consequência "Prazer de Dirigir" que, por sua vez, ligou-se 3 vezes diretamente e uma indiretamente ao valor "Autoestima/Ego". Já o atributo "Econômico" obteve 3 ligações diretas e uma indireta com a consequência "Menor manutenção/Prevenir estrago"; observando-se a matriz, é perceptível que essa consequência está relacionada Io vezes diretamente e uma vez indiretamente com outra consequência, "Sobrar mais dinheiro", a qual está ligada diretamente i2 vezes com a consequência "Lazer”. Enfim, o "Lazer” está conectado 2 vezes diretamente e uma vez indiretamente ao valor "Tranquilidade", o qual está relacionado 3 vezes diretamente com o valor "Felicidade".

Essas relações podem ser melhor compreendidas quando apresentadas no mapa hierárquico de valor, o qual esquematiza as conexões entre os atributos, as consequências e os valores. Assim, é possível visualizar que o atributo "Econômico" possui ligações diretas com a consequência "Menor manutenção/Prevenir estrago", mas ligações indiretas com a consequência "Lazer". Como também, que a consequência "Conforto" apresenta ligações indiretas com o valor "Felicidade" e assim por diante.

É importante ressaltar que neste estudo, conexões como as que existem entre os atributos "Acabamento Interno" e a consequência "Conforto" foram desconsideradas para a elaboração do mapa, pois alcançaram somente uma ligação direta, sendo necessárias, pelo menos, 3 ligações como é explicado no item 4.2, "Mapa hierárquico de valor". 
Tabela I - Matriz de Implicação

Revista Administração em Diálogo

ISSN 2178-0080

Programa de Estudos Pós-Graduados em Administração

Pontifícia Universidade Católica de São Paulo 
Tayanne Ferraz da Silva Poberschnigg, Márcio Lopes Pimenta. RAD Vol.17, n.3, Set/Out/Nov/Dez 2015, p.59-86.

\begin{tabular}{|c|c|c|c|c|c|c|c|c|c|c|c|c|c|c|c|c|c|c|c|c|c|c|c|c|c|c|c|c|c|c|c|c|c|c|c|c|}
\hline & 0,0 & {$[0,0]$} & 0,0 & {$[0,0]$} & $J_{, 0}$ & \begin{tabular}{|l|l|} 
\\
\end{tabular} & \begin{tabular}{|l|l|} 
& 0,0 \\
\end{tabular} & {$[0,0]$} & {$[0,0] 0$} & \begin{tabular}{l|l}
0,0 \\
\end{tabular} & 0,0 & 0,0 & 0,6 & 0,0 & {$[0,0]$} & {$[0,0] 0$} & {$[0,0] 0$} & '0,0 & 1,0 & '0,0 & 0,0 & 0,0 & 0,0 & $0,1]$ & $0,0 \mid$ & \begin{tabular}{l|l} 
\\
\end{tabular}, 0,0 & 0,0 & $0,0 \mid '$ & \begin{tabular}{|l|l|} 
\\
\end{tabular} & {$[0,0]$} & $' 0,1$ & '0,1|' & \begin{tabular}{l|l}
0,0 & 0 \\
\end{tabular} & 0,0 & \begin{tabular}{l|l|l}
0,0 & 0 \\
\end{tabular} & \\
\hline parelho de Som & $\mid 0,0$ & 0,0 & $\mid 0,0$ & $\mid 0,0$ & 0,0 & '0,0|' & $\begin{array}{l}0,0 \mid '( \\
\end{array}$ & $\begin{array}{l}0,0 \mid '(1 \\
\end{array}$ & \begin{tabular}{l|l}
0,0 & 10 \\
\end{tabular} & \begin{tabular}{l|l}
0,0 & 0 \\
\end{tabular} & $10,\left.0\right|^{\prime \prime}$ & \begin{tabular}{l|l}
0,0 & 0 \\
\end{tabular} & 0,0 & 0,0 & \begin{tabular}{|l|l|} 
& 0,0 \\
\end{tabular} & $\begin{array}{l}0,0 \mid c \\
\end{array}$ & \begin{tabular}{l|l}
0,0 & 10 \\
\end{tabular} & $\begin{array}{l}0,0 \\
\end{array}$ & 1,0 & $|0,0|$ & '0,0|' & $' 0,0$ & $|0,0|$ ' & \begin{tabular}{l|l}
0,0 & 10 \\
\end{tabular} & $0,0 \mid$ & \begin{tabular}{l|l}
0,0 & $\prime 0$ \\
\end{tabular} & 0,0 & \begin{tabular}{|l|l|} 
& $0,0 \mid '($
\end{tabular} & \begin{tabular}{|l|l|} 
\\
\end{tabular} & $\begin{array}{l}0,0 \\
0,1\end{array}$ & '0,0 & $|' 0,0| '(\mathrm{~s}$ & \begin{tabular}{l|l}
0,0 & 0 \\
\end{tabular} & 0,1 & \begin{tabular}{l|l|l}
0,0 & 10
\end{tabular} & \\
\hline Bluetooth & 10,0 & 10,0 & $\mid 0,0$ & $\mid 0,0$ & 0,0 & \begin{tabular}{|l|l|}
$' 0,0$ & ' \\
\end{tabular} & \begin{tabular}{|l|l|}
$' 0,0$ & ' \\
\end{tabular} & \begin{tabular}{|l|l|}
$\prime 0,0$ & 1 \\
\end{tabular} & \begin{tabular}{|l|l|}
0,0 & 0 \\
\end{tabular} & \begin{tabular}{l|l}
0,0 & 0 \\
\end{tabular} & '0,0' (' & \begin{tabular}{l|l}
0,0 & 10 \\
\end{tabular} & 0,0 & 0,0 & \begin{tabular}{|l|l|}
$' 0,0$ & ' \\
\end{tabular} & \begin{tabular}{|l|c|}
0,0 & 1 \\
\end{tabular} & \begin{tabular}{|l|l|}
0,0 & 0 \\
\end{tabular} & \begin{tabular}{l|l|l}
0,0 & 1 \\
\end{tabular} & 1,0 & $|0,0|$ & $|0,0|$ & 10,0 & \begin{tabular}{|c|c|}
$\prime$ \\
\end{tabular} & 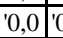 & 0,0 & \begin{tabular}{l|l}
0,0 & 0 \\
\end{tabular} & 0,0 & \begin{tabular}{|l|l|}
$' 0,0$ & ' \\
\end{tabular} & \begin{tabular}{|l|l|}
$\prime$ \\
\end{tabular} & 0 & 0 & '0,0|' & \begin{tabular}{|l|l|l} 
\\
\end{tabular}, 0,0 & 0,0 & 0 & \\
\hline uspensão Robusta & 0,0 & 0,0 & 0,0 & 0,0 & 0,0 & $\mid 0,0$ & \begin{tabular}{|l|l|} 
& \\
\end{tabular} & \begin{tabular}{|l|l|}
0,0 & \\
\end{tabular} & \begin{tabular}{ll|l}
0,0 & 0 \\
\end{tabular} & \begin{tabular}{l|l}
0,0 & 0 \\
\end{tabular} & 10,0 & \begin{tabular}{l|l}
0,0 & 0 \\
0
\end{tabular} & 0,0 & 0,0 & \begin{tabular}{|l|l|} 
& \\
\end{tabular} & \begin{tabular}{|l|l|}
0,0 & 1 \\
\end{tabular} & \begin{tabular}{ll|l}
0,0 & 0 \\
\end{tabular} & \begin{tabular}{l|l}
0,0 & 1 \\
\end{tabular} & 1,0 & '0,1' & $' 1,2$ & 2,0 & $0,0 \quad$ & 910 & 0,0 & \begin{tabular}{l|l}
0,0 & 0 \\
\end{tabular} & 0,2 & \begin{tabular}{l|l}
0,0 & 1 \\
\end{tabular} & '0,0 & 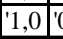 & 0,2 & 0,2 & \begin{tabular}{l|l|}
0,0 & 1 \\
\end{tabular} & 1,3 & 0,0 & \\
\hline Desenho-Ap & '0,0 & 0,0 & 0,0 & 0,0 & 0,0 & '0,0|' & \begin{tabular}{|c|c|}
0,0 \\
\end{tabular} & \begin{tabular}{|l|c|}
0,0 & 1 \\
\end{tabular} & $\begin{array}{l}0,0 \\
0\end{array}$ & \begin{tabular}{l|l}
0,0 \\
0
\end{tabular} & 0,0 ' & \begin{tabular}{l|l}
0,0 & 10 \\
0
\end{tabular} & 0,0 & 0,0 & \begin{tabular}{|c|c|}
0,0 \\
\end{tabular} & \begin{tabular}{|l|l|l|}
0,0 & $\mathrm{C}$ \\
\end{tabular} & $\begin{array}{l}0,0 \\
0\end{array}$ & $\begin{array}{l}5,0 \\
5\end{array}$ & 2,0 & $\mid 0,0$ & '0,0' & 0,0 & '0,0|' & \begin{tabular}{ll|l}
0,1 & $\mathrm{C}$ \\
\end{tabular} & (0,0)' & $\begin{array}{l} \\
0,1\end{array}$ & 1,0 & \begin{tabular}{|c|c|}
1,3 \\
\end{tabular} & '0,0|' & \begin{tabular}{|l|l|}
1,2 \\
\end{tabular} & 0,1 & $\begin{array}{ll}0,3 \\
\end{array}$ & $\begin{array}{l}0,0 \\
0\end{array}$ & 1,1 & 0,0 & \\
\hline conomico & 0,0 &, 0 & 0,0 & 0,0 & 0,0 & \begin{tabular}{|l|l|} 
\\
\end{tabular} & \begin{tabular}{|l|l} 
& \\
\end{tabular} & \begin{tabular}{|l|l|}
$1,1,0$ \\
\end{tabular} & \begin{tabular}{|l|} 
\\
\end{tabular} & $\begin{array}{l}0,0 \\
0\end{array}$ & 0,0 & \begin{tabular}{l|l}
0,0 & 0 \\
0
\end{tabular} & 0,0 & 0,0 & \begin{tabular}{|l|l|} 
& \\
\end{tabular} & \begin{tabular}{|l|l|}
$0,0,3$ \\
\end{tabular} & 18 & $\begin{array}{l}0,0 \\
0\end{array}$ & 0,0 & 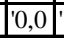 & \begin{tabular}{|l|l|} 
\\
\end{tabular} & \begin{tabular}{|l|} 
\\
\end{tabular} 11,4 & \begin{tabular}{|l|l|} 
\\
\end{tabular} & 7 & 0,1 & $\begin{array}{l}0,1 \\
0,1\end{array}$ & 0,9 & 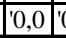 & \begin{tabular}{|l|} 
\\
\end{tabular} & 03 & & ' & \begin{tabular}{|l|l}
0,1 & 0 \\
\end{tabular} & 0,10 & & \\
\hline sspaço i & \begin{tabular}{|l|l|l}
0 & 0,0 \\
\end{tabular} & 0,0 & $\mid 0,0$ & $\mid$ & 0,0 & \begin{tabular}{|l|l|} 
\\
\end{tabular} & \begin{tabular}{|l|l|} 
\\
\end{tabular} & \begin{tabular}{|c|c|} 
\\
\end{tabular} & \begin{tabular}{l|l|} 
\\
\end{tabular} & $\begin{array}{l}0,0 \\
\end{array}$ & '0,0' & \begin{tabular}{l|l}
0,0 & 0 \\
\end{tabular} & 0,0 & 0,0 & \begin{tabular}{|c|c|} 
\\
\end{tabular} & \begin{tabular}{|l|l} 
\\
\end{tabular} & \begin{tabular}{|l|l} 
\\
\end{tabular} & \begin{tabular}{l|l}
0,0 & ', \\
\end{tabular} & 9,0 & \begin{tabular}{|l|l|} 
\\
\end{tabular} & '0,0' & \begin{tabular}{|l|} 
\\
\end{tabular} & \begin{tabular}{|c|} 
\\
\end{tabular} & \begin{tabular}{|l|}
0,4 \\
\end{tabular} & 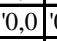 & $\begin{array}{l}0,1 \\
0,1\end{array}$ & 0,0 & \begin{tabular}{|c|c|} 
\\
\end{tabular} & \begin{tabular}{|l|l|} 
\\
\end{tabular} & \begin{tabular}{l|l}
0,0 \\
\end{tabular} & ),2 & 0,2 & \begin{tabular}{|l|l}
0,0 & 10 \\
\end{tabular} & 0,5 & 0,0 & $\overline{0,0}$ \\
\hline ens & \begin{tabular}{|l|} 
\\
\end{tabular} & 0,0 & 0,0 & \begin{tabular}{|l|}
0,0 \\
\end{tabular} & 0,0 & \begin{tabular}{|l|l|} 
\\
\end{tabular} & \begin{tabular}{|l|l|} 
& \\
\end{tabular} & \begin{tabular}{|l|l|} 
& \\
\end{tabular} & $\begin{array}{l}0,0 \\
0\end{array}$ & $\begin{array}{l}0,0 \\
0\end{array}$ & $10,0 \mid$ & \begin{tabular}{l|l}
0,0 & 1 \\
0
\end{tabular} & 0,0 & 0,0 & \begin{tabular}{|l|l|} 
& \\
\end{tabular} & \begin{tabular}{|l|l|} 
& \\
\end{tabular} & \begin{tabular}{|c|c}
0,0 \\
\end{tabular} & \begin{tabular}{l|l}
0,3 & 1
\end{tabular} & '11,0' & \begin{tabular}{|l|l} 
\\
\end{tabular} & \begin{tabular}{|l|l|} 
\\
\end{tabular} & 0,0 & '0,1' & \begin{tabular}{|l|l}
3,2 & \\
\end{tabular} & 0,0 & \begin{tabular}{l|l}
0,0 & \\
0
\end{tabular} & 0,1 & \begin{tabular}{|c|c|} 
\\
\end{tabular} & \begin{tabular}{|l|l|} 
\\
\end{tabular} & \begin{tabular}{|l|l|} 
& \\
\end{tabular} & & \begin{tabular}{|l|l|}
0,2 & \\
\end{tabular} & \begin{tabular}{|l|l}
0,0 & 0 \\
\end{tabular} & & & \\
\hline ens & 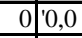 &, 0 & 0,0 & 0 & 0,0 & '0,0|' & '|'|'| & \begin{tabular}{|c|c|} 
& \\
\end{tabular} & $\begin{array}{l}0,0 \\
\end{array}$ & $\begin{array}{l}0,0 \\
0\end{array}$ & 0,0 & \begin{tabular}{l|l}
0,0 & 1 \\
\end{tabular} & $0,0,0$ & 0,0 & '|' & \begin{tabular}{|l|l|} 
\\
\end{tabular} & \begin{tabular}{|l|l|}
2,0 \\
\end{tabular} & $\begin{array}{l}0,0 \\
0,0\end{array}$ & 1,0 & $|0,0|$ & \begin{tabular}{|c|} 
\\
\end{tabular} & '0,1 & '0,1' & \begin{tabular}{l|l|l} 
\\
\end{tabular} & '0,1' & $\begin{array}{l}0,0 \\
0,0\end{array}$ & 0,0 & \begin{tabular}{|c|c|} 
\\
\end{tabular} & \begin{tabular}{|l|} 
\\
\end{tabular} & 0,0 & 5 & 0 & \begin{tabular}{l|l}
0,0 & 0 \\
\end{tabular} & 0,1 & 0,0 & \\
\hline arca & \begin{tabular}{c|c|c|}
$0,0,0$ \\
\end{tabular} & 0,0 & $\mid 0,0$ & $0,0,0$ & 0,0 & \begin{tabular}{|l|l|} 
\\
\end{tabular} & \begin{tabular}{|l|l|} 
& \\
\end{tabular} & \begin{tabular}{|l|l|} 
& \\
\end{tabular} & \begin{tabular}{|c|c} 
\\
\end{tabular} & \begin{tabular}{l|l}
0,0 & 0
\end{tabular} & '0,0' & \begin{tabular}{l|l}
1,0 & \\
\end{tabular} & 0,0 & 0,0 & \begin{tabular}{|l|l|}
6,1 & \\
\end{tabular} & \begin{tabular}{|l|l|}
4,1 & \\
\end{tabular} & \begin{tabular}{|l|l|}
4,2 & \\
\end{tabular} & \begin{tabular}{l|l}
0,0 & 1 \\
\end{tabular} & 1,1 & $\left|{ }^{\prime} 0,0\right|^{\prime}$ & $' 0,3 \mid$ & $\begin{array}{l} \\
\end{array}$ & \begin{tabular}{|l|l|} 
\\
\end{tabular} & \begin{tabular}{|l|l|}
2,7 & \\
\end{tabular} & '0,0' & $\begin{array}{l}0,0 \\
0\end{array}$ & 0,1 & \begin{tabular}{|l|l|} 
\\
\end{tabular} & \begin{tabular}{|l|l|} 
\\
\end{tabular} & \begin{tabular}{l|l|}
0,1 & \\
\end{tabular} & L & '0,5' & \begin{tabular}{|l|l}
0,0 & 0 \\
\end{tabular} & 0,4 & \begin{tabular}{|l|l}
0,0 & 0 \\
\end{tabular} & 0,1 \\
\hline 00 & \begin{tabular}{|l|} 
\\
\end{tabular} & 0 & $\mid 0,0$ &, 0 & 20,0 & ' & \begin{tabular}{|l|l|} 
& \\
\end{tabular} & \begin{tabular}{|l|l|} 
& \\
\end{tabular} & '0,0 & \begin{tabular}{l|l}
0,0 & 0 \\
\end{tabular} & ' & \begin{tabular}{l|l}
0,0 & 1
\end{tabular} & 0,0 & 0,0 & \begin{tabular}{|l|l|} 
& \\
\end{tabular} & \begin{tabular}{|l|l|} 
& \\
\end{tabular} & \begin{tabular}{|l|l|}
1,0 & \\
\end{tabular} & \begin{tabular}{|l|l}
2,0 & 0
\end{tabular} & 0,1 & ||$^{\prime} 0,0 \mid$ & $|'| 1,1 \mid$ & \begin{tabular}{|l|} 
\\
\end{tabular} & \begin{tabular}{|l|} 
\\
\end{tabular} & & '0,1' & $\begin{array}{l}0,0 \\
\end{array}$ & 0,0 & \begin{tabular}{|l|l|} 
\\
\end{tabular} & \begin{tabular}{|l|} 
\\
\end{tabular} &, 4 & & '0,1' & \begin{tabular}{|l|l|} 
\\
\end{tabular} & 0,3 & 0,0 & \\
\hline eço & \begin{tabular}{|c|c|c|}
0 & 0,0 \\
\end{tabular} & 0 & 0,0 & 0,0 & 0,0 & \begin{tabular}{|l|l|} 
\\
\end{tabular} & '|' & \begin{tabular}{|c|c|} 
& \\
\end{tabular} & 0,0 & $\begin{array}{l}, 0 \\
, 0\end{array}$ & '0,0' & \begin{tabular}{l|l}
0,0 & 1 \\
\end{tabular} & 1,0 & 2,0 & ' & \begin{tabular}{|l|l|} 
& 10,0 \\
\end{tabular} & \begin{tabular}{l|l|} 
\\
\end{tabular} & \begin{tabular}{l|l}
0,1 & 2 \\
\end{tabular} & 2,1 & $|0,0|$ & '0,1' & $4,4,3$ & \begin{tabular}{|l|} 
\\
\end{tabular} & \begin{tabular}{l|l|}
0,5 & \\
\end{tabular} & 2,1 & \begin{tabular}{l|l}
0,0 & 1
\end{tabular} & 0,1 & \begin{tabular}{|l|l|} 
\\
\end{tabular} & \begin{tabular}{|l|} 
\\
\end{tabular} & 02 & ,2 & '0,3' & \begin{tabular}{|l|l}
0,0 & 0 \\
\end{tabular} & 0,6 & 0,1 & \\
\hline esis & \begin{tabular}{|l|l|l}
0 & 0,0 \\
\end{tabular} &, 0 & $\mid 0,0$ & 0,0 & 0,0 & \begin{tabular}{|l|l|} 
\\
\end{tabular} & \begin{tabular}{|l|l|} 
& \\
\end{tabular} & \begin{tabular}{|c|c|} 
& \\
\end{tabular} & \begin{tabular}{l|l} 
\\
\end{tabular} & \begin{tabular}{l|l}
0,0 & 1
\end{tabular} & ' & $\begin{array}{l}0,0 \\
0,0\end{array}$ & 0,0 & 0,0 & \begin{tabular}{|l|l|} 
& \\
\end{tabular} & \begin{tabular}{|l|l|}
0,0 & \\
\end{tabular} & $\begin{array}{l}5,0 \\
5\end{array}$ & \begin{tabular}{l|l}
0,0 \\
\end{tabular} & 0,0 & $' 0,0$ ' & $10,\left.0\right|^{\prime}$ & 0,4 & ||$^{\prime} 0,0 \mid$ & \begin{tabular}{|l|}
3,1 \\
\end{tabular} & 0,0 & \begin{tabular}{l|l}
0,0 & 1 \\
0
\end{tabular} & 0,3 & '0,1' & \begin{tabular}{|l|} 
\\
\end{tabular} & \begin{tabular}{|l|l}
0,3 \\
\end{tabular} & '0,1 & ' ' & \begin{tabular}{|l|l}
0,0 & 0 \\
\end{tabular} & 0,4 & \begin{tabular}{l|l}
0,0 & 0 \\
\end{tabular} & 0,0 \\
\hline & \begin{tabular}{|c|c|c|}
0 & 0,0 \\
\end{tabular} & 0 & 0,0 & 0,0 & 0,0 & \begin{tabular}{|l|l|} 
\\
\end{tabular} & \begin{tabular}{|l|l|} 
& \\
\end{tabular} & \begin{tabular}{|c|c|} 
& \\
\end{tabular} & '0,0 & $\begin{array}{l}, 0 \\
, 0\end{array}$ & '0,0" & \begin{tabular}{l|l}
0,0 & 1 \\
\end{tabular} & 0,0 & 0,0 & \begin{tabular}{|l|l|} 
& \\
\end{tabular} & \begin{tabular}{|c|c|} 
& \\
\end{tabular} & \begin{tabular}{l|l}
0,0 \\
\end{tabular} & \begin{tabular}{l|l}
0,0 & 1 \\
\end{tabular} & 1,0 & $|0,0|$ & \begin{tabular}{|l|} 
\\
\end{tabular} & \begin{tabular}{|l|} 
\\
\end{tabular} & \begin{tabular}{|l|} 
\\
\end{tabular} & $\begin{array}{l}0,1 \\
\end{array}$ & '0,0' & \begin{tabular}{l|l}
0,0 & 1
\end{tabular} & 0,0 & \begin{tabular}{|l|l|} 
\\
\end{tabular} & \begin{tabular}{|l|} 
\\
\end{tabular} & & & 0 & '0 & 0,1 & & \\
\hline & \begin{tabular}{|l|l|l}
0 & 10,0 \\
\end{tabular} & 0,0 & $\mid 0,0$ & $|0,0|$ & 0,0 & \begin{tabular}{|l|l|} 
\\
\end{tabular} & \begin{tabular}{|l|l|} 
& \\
\end{tabular} & \begin{tabular}{|l|l|} 
& \\
\end{tabular} & \begin{tabular}{l|l|l} 
& 0,0 \\
\end{tabular} & $\begin{array}{l}0,0 \\
0\end{array}$ & ' & $\begin{array}{l}0,0 \\
0,0\end{array}$ & 0,0 & 0,0 & \begin{tabular}{|c|c|} 
& \\
\end{tabular} & \begin{tabular}{|l|l|} 
& 10,0 \\
\end{tabular} & \begin{tabular}{l|l}
1,0 \\
\end{tabular} & \begin{tabular}{l|l}
0,0 \\
0
\end{tabular} & 0,0 & \begin{tabular}{|c|} 
\\
\end{tabular} & \begin{tabular}{|l|l|} 
\\
\end{tabular} & $\begin{array}{l}1,0 \\
\end{array}$ & '0,1' & \begin{tabular}{|c|c} 
\\
\end{tabular} & '0,0' & \begin{tabular}{l|l}
0,0 & 1 \\
0
\end{tabular} & 0,0 & \begin{tabular}{|c|c|} 
\\
\end{tabular} & $|0,0|$ & \begin{tabular}{|l|} 
\\
\end{tabular} & 1 & 0,0 & \begin{tabular}{|l|l}
0,0 & 0 \\
\end{tabular} & 0,0 & 0,1 & $\overline{0,0}$ \\
\hline rradiç̧a & \begin{tabular}{|c|c|c|}
0 & 10,0 \\
\end{tabular} & 0,0 & 0,0 & 0,0 & 0,0 & \begin{tabular}{|l|l|} 
\\
\end{tabular} & \begin{tabular}{|l|l|} 
& \\
\end{tabular} & \begin{tabular}{|c|c|} 
& \\
\end{tabular} & \begin{tabular}{l|l}
0,0 \\
\end{tabular} & \begin{tabular}{l|l}
0,0 & 0 \\
0
\end{tabular} & '0,0 ' & \begin{tabular}{l|l}
0,0 & 1 \\
\end{tabular} & 0,0 & 0,0 & \begin{tabular}{|l|l|} 
\\
\end{tabular} & \begin{tabular}{|l|l|}
11,0 & \\
\end{tabular} & \begin{tabular}{|l|l|}
2,0 \\
\end{tabular} & \begin{tabular}{l|l}
0,0 & 0 \\
\end{tabular} & 0,0 & $|0,0|$ & \begin{tabular}{|l|} 
\\
\end{tabular} & \begin{tabular}{|l|} 
\\
\end{tabular} & '0,1' & \begin{tabular}{|l|l|}
2,2 & \\
\end{tabular} & 0,0 & \begin{tabular}{l|l}
0,0 & 1
\end{tabular} & 0,0 & \begin{tabular}{|l|l|} 
\\
\end{tabular} & '2,0|' & \begin{tabular}{|l|l|} 
& \\
\end{tabular} & 0,5 & \begin{tabular}{|c|c|} 
\\
\end{tabular} & \begin{tabular}{|l|l|l}
0,0 & 1 \\
\end{tabular} & 1,0 & \begin{tabular}{|l|l}
0,0 & 0 \\
\end{tabular} & $\overline{0,0}$ \\
\hline Ater & \begin{tabular}{|l|l|l}
0 & 10,0 \\
\end{tabular} & 0 & 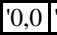 & 0 & 0,0 & \begin{tabular}{|l|l|} 
\\
\end{tabular} & \begin{tabular}{|l|l|} 
& \\
\end{tabular} & \begin{tabular}{|l|l|} 
& \\
\end{tabular} & 0,0 & \begin{tabular}{l|l}
, 0 & 0 \\
\end{tabular} & 0,0 & $\begin{array}{l}0,0 \\
0,0\end{array}$ & 0,0 & 0,0 & \begin{tabular}{|l|l|} 
& \\
\end{tabular} & \begin{tabular}{|l|l|} 
& \\
\end{tabular} & \begin{tabular}{l|l|l} 
& 0,0 \\
\end{tabular} & \begin{tabular}{l|l|}
0,0 & \\
\end{tabular} & 0,0 & '0,0|' & $1,1,0$ & 0,0 & '0,1' & 2,0 & 0,0 & \begin{tabular}{l|l}
0,0 & \\
\end{tabular} & 0,0 & '|' & '0,1 & 0,1 & 1,0 & 0,3 & \begin{tabular}{|l|l}
0,0 & 0 \\
\end{tabular} & 0,0 & & \\
\hline Men & \begin{tabular}{|c|c|c|}
0 & 0,0 \\
\end{tabular} & 0 & 10,0 & 0,0 & 0,0 & \begin{tabular}{|l|l|} 
\\
\end{tabular} & \begin{tabular}{|l|l|} 
& \\
\end{tabular} & \begin{tabular}{|c|c|} 
& \\
\end{tabular} & \begin{tabular}{l|l}
0,0 \\
\end{tabular} & \begin{tabular}{l|l}
0,0 & 0 \\
0
\end{tabular} & '0,0 'ו & \begin{tabular}{l|l}
0,0 & 0 \\
\end{tabular} & 0,0 & 0,0 & \begin{tabular}{|l|l|} 
& \\
\end{tabular} & \begin{tabular}{|c|c|} 
& \\
\end{tabular} & \begin{tabular}{l|l}
0,0 \\
\end{tabular} & \begin{tabular}{l|l}
0,0 & 1 \\
\end{tabular} & 1,0 & $|'| c, 0 \mid$ & \begin{tabular}{|c|} 
\\
\end{tabular}, 0,0 & \begin{tabular}{|l|} 
\\
\end{tabular} & \begin{tabular}{|l|l|} 
& \\
\end{tabular} & \begin{tabular}{|l|l|}
3,3 & \\
\end{tabular} & '0,0' & \begin{tabular}{l|l}
0,0 & 1
\end{tabular} & 1,5 & \begin{tabular}{|l|l|} 
\\
\end{tabular} & \begin{tabular}{|l|l|} 
& $1,1,0$ \\
\end{tabular} & \begin{tabular}{|l|l|} 
& \\
\end{tabular} & '0,4 & '0,3' & \begin{tabular}{|l|l}
0,0 & 0 \\
\end{tabular} & 0,8 & \begin{tabular}{|l|l}
0,0 & 0 \\
\end{tabular} & 0,0 \\
\hline Praze & \begin{tabular}{c|c|c}
0 & 0,0 \\
\end{tabular} & \begin{tabular}{|c|} 
\\
\end{tabular} & $\mid 0,0$ & \begin{tabular}{|c|} 
\\
\end{tabular} & 0,0 & ' & \begin{tabular}{|l|l|} 
& \\
\end{tabular} & \begin{tabular}{|l|l|} 
& \\
\end{tabular} & \begin{tabular}{|l|l|} 
\\
\end{tabular} & \begin{tabular}{l|l}
0,0 & \\
\end{tabular} & '0,0 ' & \begin{tabular}{l|l}
0,0 & 1
\end{tabular} & 0,0 & 0,0 & \begin{tabular}{|l|l|} 
& \\
\end{tabular} & \begin{tabular}{|l|l|} 
& \\
\end{tabular} & \begin{tabular}{|l|l} 
\\
\end{tabular} & \begin{tabular}{l|l}
0,0 & \\
\end{tabular} & 2,0 & \begin{tabular}{|l|l|} 
\\
\end{tabular} & \begin{tabular}{|l|l|} 
\\
\end{tabular} & \begin{tabular}{|l|} 
\\
\end{tabular} & $|'|{ }^{\prime \prime}$ & \begin{tabular}{|l|l|}
2,0 & \\
\end{tabular} & '0,0' & $\begin{array}{l} \\
0,0\end{array}$ & '0,1 & '3,1' & \begin{tabular}{|l|l|} 
& \\
\end{tabular} & \begin{tabular}{|l|l|}
2,2 & 1 \\
\end{tabular} & 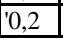 & \begin{tabular}{|c|c|} 
& \\
\end{tabular} & \begin{tabular}{|l|l|l}
0,0 & 2 \\
\end{tabular} & 2,2 & \begin{tabular}{|l|l}
0,0 & 0 \\
\end{tabular} & $\overline{0,0}$ \\
\hline$n$ & \begin{tabular}{r|l}
$0,0,0$ \\
\end{tabular} & $\mid{ }^{\prime} 0,0,{ }^{\prime}$ & $\mid 0,0$ & 0,0 & 0,0 & \begin{tabular}{|l|l|} 
\\
\end{tabular} & \begin{tabular}{|l|l|} 
& \\
\end{tabular} & \begin{tabular}{|c|c|} 
& 10,0 \\
\end{tabular} & $\begin{array}{l} \\
\end{array}$ & $\begin{array}{l}0,0 \\
\end{array}$ & 0,0 & $\begin{array}{l}0,0 \\
0,0\end{array}$ & 0,0 & 0,0 & \begin{tabular}{|l|l|} 
\\
\end{tabular} & \begin{tabular}{|l|l|} 
& 10,0 \\
\end{tabular} & $0,0]^{3}$ & \begin{tabular}{|l|l|}
3,0 & \\
\end{tabular} & 0 & \begin{tabular}{|c|} 
\\
\end{tabular} & '1,1' & 2,0 & \begin{tabular}{|c|} 
\\
\end{tabular} & 6,2 & '0,0 & $\begin{array}{l}0,1 \\
\end{array}$ & 0,1 & \begin{tabular}{|l|}
2,1 \\
\end{tabular} & 2,1 & 2,3 & 6 & 0,4 & \begin{tabular}{|l|l|l|}
0,0 & \\
\end{tabular} & 9,2 & 0,0 & 0,0 \\
\hline ev & \begin{tabular}{c|c|c|}
$0,0,0$ \\
\end{tabular} & $0^{\prime}$ & $\mid 0,0$ & \begin{tabular}{|c|} 
\\
\end{tabular} & 0,0 & \begin{tabular}{|l|l|} 
\\
\end{tabular} & \begin{tabular}{|l|} 
\\
\end{tabular} & \begin{tabular}{|l|l|} 
& \\
\end{tabular} & \begin{tabular}{|l|l} 
& \\
\end{tabular} & \begin{tabular}{l|l}
0,0 & 0
\end{tabular} & 10,0 & \begin{tabular}{l|l}
0,0 & 0 \\
0
\end{tabular} & 0,0 & 0,0 & \begin{tabular}{|l|} 
\\
\end{tabular} & \begin{tabular}{|l|l|} 
& \\
\end{tabular} & \begin{tabular}{|l|l} 
& \\
\end{tabular} & \begin{tabular}{l|l}
0,0 & \\
\end{tabular} & 0,0 & 10,0 & '0,0' & \begin{tabular}{|l|} 
\\
\end{tabular} & \begin{tabular}{|l|l|} 
\\
\end{tabular} & \begin{tabular}{|l|l} 
& \\
\end{tabular} & '0,0' & \begin{tabular}{l|l}
1,0 & 1
\end{tabular} & 1,0 & 10,0 ' & \begin{tabular}{|l|l|} 
\\
\end{tabular} & \begin{tabular}{|l|l|} 
& \\
\end{tabular} & 0,1 & ' & \begin{tabular}{l|l}
0,0 & 0 \\
\end{tabular} & 0,0 & $\begin{array}{l}0,0 \\
\end{array}$ & 0,0 \\
\hline Econo & \begin{tabular}{|l|l|l}
0 & 0,0 \\
\end{tabular} & \begin{tabular}{|c|} 
\\
\end{tabular} & $\mid 0,0$ & $\mid 0,0$ & 0,0 & \begin{tabular}{|l|l|} 
\\
\end{tabular} & \begin{tabular}{|l|l|} 
& \\
\end{tabular} & \begin{tabular}{|l|l|} 
& 0,0 \\
\end{tabular} & \begin{tabular}{l|l|l} 
& 0,0 \\
\end{tabular} & $\begin{array}{l}0,0 \\
\end{array}$ & ' & \begin{tabular}{l|l}
0,0 & 0 \\
\end{tabular} & 0,0 & 0,0 & \begin{tabular}{|l|l|} 
& \\
\end{tabular} & \begin{tabular}{|c|c|} 
& \\
\end{tabular} & \begin{tabular}{l|l|l} 
& 0,0 \\
\end{tabular} & \begin{tabular}{l|l|}
0,0 & \\
\end{tabular} & 0 & $|'| 1,0 \mid$ & \begin{tabular}{|c|} 
\\
\end{tabular} & 0,1 & \begin{tabular}{|l|} 
\\
\end{tabular} & '1, '1, ' & $1,1,2$ & $\begin{array}{l} \\
0,0\end{array}$ & 1,1 & $|0,0|$ & \begin{tabular}{|l|l|} 
\\
\end{tabular} & \begin{tabular}{|l|} 
\\
\end{tabular} & 1,3 & \begin{tabular}{|l|l|} 
\\
\end{tabular} & \begin{tabular}{l|l|l}
0,0 & 2 \\
\end{tabular} & 2,3 & 0,0 & 00 \\
\hline & \begin{tabular}{c|c|c|}
$0,0,0$ \\
\end{tabular} & \begin{tabular}{|l|l|} 
\\
\end{tabular} & 0,0 & \begin{tabular}{|c|} 
\\
\end{tabular} & 0,0 & \begin{tabular}{|l|l|} 
\\
\end{tabular} & \begin{tabular}{|l|l|} 
& \\
\end{tabular} & \begin{tabular}{|l|l|} 
& \\
\end{tabular} & \begin{tabular}{|l|l}
0,0 & \\
\end{tabular} & \begin{tabular}{l|l}
0,0 & \\
\end{tabular} & $0,0,0$ & \begin{tabular}{l|l}
0,0 & 0 \\
\end{tabular} & 0,0 & 0,0 & \begin{tabular}{|l|l|} 
& \\
\end{tabular} & \begin{tabular}{|l|l|} 
& 0,0 \\
\end{tabular} & \begin{tabular}{l|l}
0,0 & \\
\end{tabular} & $\begin{array}{l}0,0 \\
0,0\end{array}$ & 0,0 & \begin{tabular}{|l|l|} 
\\
\end{tabular} & $|'|, 0 \mid$ & $\begin{array}{l} \\
\end{array}$ & $1,1,0$ & $3,1] 1$ & '1,0' & $\begin{array}{l}1,0 \\
1,0\end{array}$ & 12,0 & $|0,0|$ & \begin{tabular}{|l|l|}
$1,1,0$ \\
\end{tabular} & \begin{tabular}{|l|l|}
3,3 \\
\end{tabular} & & \begin{tabular}{|c|c|} 
& \\
\end{tabular} & \begin{tabular}{|l|l|l}
0,1 & 6 \\
\end{tabular} & 6,6 & \begin{tabular}{|l|l}
0,1 & 0 \\
\end{tabular} & 0,0 \\
\hline 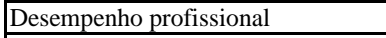 & \begin{tabular}{|c|c|c|}
0 & 1 \\
\end{tabular} & \begin{tabular}{|c|} 
\\
\end{tabular} & 0,0 & 0,0 & 0,0 & '0,0 & \begin{tabular}{|c|} 
\\
\end{tabular} & \begin{tabular}{|c|c|} 
& \\
\end{tabular} & $\begin{array}{l}0,0 \\
\end{array}$ & $\begin{array}{l}0,0 \\
\end{array}$ & '0,0' & \begin{tabular}{l|l}
0,0 & 1 \\
\end{tabular} & 0,0 & 0,0 & '|' & \begin{tabular}{|c|c|} 
\\
\end{tabular} & $\begin{array}{l}0,0 \\
\end{array}$ & $\begin{array}{l}0,0 \\
\end{array}$ & 0,0 & $' 0,0 \mid$ & \begin{tabular}{|c|} 
\\
\end{tabular} & \begin{tabular}{|l|} 
\\
\end{tabular} & '0,0|' & \begin{tabular}{l|l|l} 
\\
\end{tabular} & 2,0 & $\begin{array}{l}0,0 \\
\end{array}$ & 0,0 & '0,0' & \begin{tabular}{|l|l} 
\\
\end{tabular} & '4,0' & 2 & '1, '1,0' & \begin{tabular}{l|l}
0,0 & 0 \\
\end{tabular} & 0,1 & \begin{tabular}{|l|l|l|l|} 
\\
\end{tabular} & $\overline{0,0}$ \\
\hline Diri & \begin{tabular}{c|c|c|}
$0,0,0$ \\
\end{tabular} & , & '0,0 & \begin{tabular}{|c|} 
\\
\end{tabular} & 0,0 & \begin{tabular}{|l|l|} 
\\
\end{tabular} & \begin{tabular}{|l|l|} 
& \\
\end{tabular} & \begin{tabular}{|l|l|} 
& \\
\end{tabular} & \begin{tabular}{|c|c}
0,0 \\
\end{tabular} & \begin{tabular}{l|l}
0,0 & \\
0
\end{tabular} & 0,0 & \begin{tabular}{l|l}
0,0 & 0 \\
\end{tabular} & 0,0 & 0,0 & \begin{tabular}{|l|l|} 
& \\
\end{tabular} & \begin{tabular}{|l|l|} 
& 0,0 \\
\end{tabular} & \begin{tabular}{|l|l|} 
\\
\end{tabular} & $\begin{array}{l}1,0 \\
1,0\end{array}$ & 0,0 & \begin{tabular}{|c|} 
\\
\end{tabular} & '3,0' & \begin{tabular}{|l|} 
\\
\end{tabular} & \begin{tabular}{|l|l|} 
\\
\end{tabular} & \begin{tabular}{|l|l}
0,0 & \\
\end{tabular} & '0,1' & $\begin{array}{l}1,0 \\
1,0\end{array}$ & 0,0 & '1,1' & \begin{tabular}{|l|l|}
4,0 & \\
\end{tabular} & \begin{tabular}{|l|l|}
3,3 & \\
\end{tabular} & \begin{tabular}{|l|}
12,2 \\
\end{tabular} & \begin{tabular}{|c|c|} 
\\
\end{tabular} & \begin{tabular}{|l|l|l|}
0,0 & 4 \\
\end{tabular} & 4,3 & 0,0 & \\
\hline & \begin{tabular}{|l|l|l}
0 & 10,0 \\
\end{tabular} & \begin{tabular}{|c|} 
\\
\end{tabular} & 0,0 & 0,0 & 0,0 & '0,0|' & \begin{tabular}{|l|l|} 
\\
\end{tabular} & \begin{tabular}{|c|c|} 
& \\
\end{tabular} & $\begin{array}{l}0,0 \\
\end{array}$ & $\begin{array}{l}0,0 \\
\end{array}$ & 0,0 & \begin{tabular}{l|l}
0,0 & 0 \\
\end{tabular} & 0,0 & 0,0 & \begin{tabular}{|l|l|} 
& \\
\end{tabular} & \begin{tabular}{|c|c|} 
\\
\end{tabular} & $\begin{array}{l}0,0 \\
\end{array}$ & $\begin{array}{l}0,0 \\
\end{array}$ & 0,0 & $' 0,0 \mid$ & $' 0,0 \mid$ & 0,0 & '0,0|' & $\begin{array}{l}1,0 \\
\end{array}$ & '0,0' & $\begin{array}{l}0,0 \\
0,0\end{array}$ & 0,0 & '0,0' & '0,0|' & \begin{tabular}{|l|} 
\\
\end{tabular} & 1,0 & $0,0]^{0}$ & \begin{tabular}{|l|l|l}
0,0 & \\
\end{tabular} & 3,1 & $\begin{array}{ll}0,1 & 0 \\
\end{array}$ & 0,0 \\
\hline ajudar : & \begin{tabular}{|c|c|c|}
0 & 0,0 \\
\end{tabular} &, 0 & 0,0 & 0,0 & 0,0 & \begin{tabular}{|l|l|} 
\\
\end{tabular} & \begin{tabular}{|l|l} 
& \\
\end{tabular} & \begin{tabular}{|l|l|} 
& \\
\end{tabular} & \begin{tabular}{l|l}
0,0, \\
\end{tabular} & \begin{tabular}{l|l}
0,0 & 0
\end{tabular} & '0,0' & \begin{tabular}{l|l}
0,0 & 1 \\
0
\end{tabular} & 0,0 & 0,0 & \begin{tabular}{|l|l} 
& \\
\end{tabular} & \begin{tabular}{|l|l} 
& \\
\end{tabular} & \begin{tabular}{l|l}
0,0, \\
\end{tabular} & $\begin{array}{l}0,0 \\
0\end{array}$ & 0 & $|0,0|$ & $' 0,0 \mid$ & $\begin{array}{l} \\
\end{array}$ & \begin{tabular}{|l|l|} 
\\
\end{tabular} & $\begin{array}{ll} & 10 \\
\end{array}$ & 100' & \begin{tabular}{l|l}
0,0 & 1
\end{tabular} & 0,0 & \begin{tabular}{|c|} 
\\
\end{tabular} & \begin{tabular}{|l|l} 
\\
\end{tabular} & $\frac{110}{110}$ & $\overline{00}$ & 11 & $\begin{array}{l}1,0 \\
\end{array}$ & 0 & & \\
\hline azer & \begin{tabular}{r|l}
$0,0,0$ \\
\end{tabular} & \begin{tabular}{|c|} 
\\
\end{tabular} & 0,0 & 0,0 & 0,0 & '0,0|' & \begin{tabular}{|l|l|} 
\\
\end{tabular} & \begin{tabular}{|l|l|} 
\\
\end{tabular} & $\begin{array}{l} \\
\end{array}$ & $\begin{array}{l}0,0 \\
\end{array}$ & '0,0' & $\begin{array}{l}0,0 \\
0\end{array}$ & 0,0 & 0,0 & \begin{tabular}{|l|l|} 
\\
\end{tabular} & \begin{tabular}{|l|c|}
0,0 \\
\end{tabular} & \begin{tabular}{|l|c|} 
\\
\end{tabular} & \begin{tabular}{l|l}
0,0 \\
\end{tabular} & 0,0 & $' 0,0 \mid$ & '0,0' & $\begin{array}{l}1,1,0 \\
\end{array}$ & \begin{tabular}{|l|l|} 
\\
\end{tabular} & ' 1,0 ' & 0,0 & \begin{tabular}{l|l}
0,0 \\
\end{tabular} & 0,0 & 10,0 & $\mid 0,0$ & $2,2,2$ & 2,1 & '1,2' & \begin{tabular}{l|l}
0,0 & 9 \\
\end{tabular} & 9,2 & $\begin{array}{l}, 0,0 \\
\end{array}$ & $\overline{0,0}$ \\
\hline & \begin{tabular}{|c|c|c|}
0 & 0,0 \\
\end{tabular} & & 0,0 & 0,0 & 0,0 & \begin{tabular}{|l|l|} 
\\
\end{tabular} & \begin{tabular}{|l|l|} 
& \\
\end{tabular} & \begin{tabular}{|l|l|} 
& \\
\end{tabular} & \begin{tabular}{l|l}
0,0 \\
\end{tabular} & $\begin{array}{l}, 0 \\
, 0\end{array}$ & '0,0' & \begin{tabular}{l|l}
0,0 & 1 \\
\end{tabular} & 0,0 & 0,0 & \begin{tabular}{|l|l} 
& \\
\end{tabular} & \begin{tabular}{|c|c|} 
& \\
\end{tabular} & \begin{tabular}{l|l}
0,0 \\
\end{tabular} & $\begin{array}{l}0,0 \\
0\end{array}$ & 0,0 & ||$^{\prime} 0,0 \mid$ & \begin{tabular}{|l|} 
\\
\end{tabular} & \begin{tabular}{|l|} 
\\
\end{tabular} & \begin{tabular}{|l|l|}
$\prime$ \\
\end{tabular} & \begin{tabular}{l|l}
0,0 \\
\end{tabular} & 0,0 & \begin{tabular}{l|l}
0,0 & 1
\end{tabular} & 0,0 & $' 0,0 \mid$ & \begin{tabular}{|l|l|} 
\\
\end{tabular} & \begin{tabular}{|l|l|}
$' 1,0$ \\
\end{tabular} & & '2,0' & \begin{tabular}{|l|l}
0,0 & 0 \\
\end{tabular} & 0,0 & \begin{tabular}{|l|l}
0,0 & 0 \\
\end{tabular} & $\overline{0,0}$ \\
\hline$a$ & \begin{tabular}{|c|c|c|}
0 & 1 \\
\end{tabular} & $' 0,0 \mid$ & 0,0 & 0,0 & 0,0 & '0,0|' & \begin{tabular}{|c|c|} 
\\
\end{tabular} & \begin{tabular}{l|l} 
\\
\end{tabular} & $\begin{array}{l}0,0 \\
\end{array}$ & \begin{tabular}{l|l}
0,0 \\
\end{tabular} & ' & \begin{tabular}{l|l}
0,0 \\
\end{tabular} & 0,0 & 0,0 & \begin{tabular}{|l|l|} 
& \\
\end{tabular} & \begin{tabular}{|l|l|} 
\\
\end{tabular} & $\begin{array}{l} \\
\end{array}$ & \begin{tabular}{l|l|l}
0,0 & 0 \\
\end{tabular} & 0,0 & $' 0,0$ & $10,0 \mid$ & $\begin{array}{l}1 \\
\end{array}$ & '0,0|' & $\begin{array}{l} \\
\end{array}$ & '0,0' & $\begin{array}{l}0,0 \\
\end{array}$ & 0,0 & '0,0 & \begin{tabular}{|c|c|} 
\\
\end{tabular} & \begin{tabular}{l|l|l}
0,0 \\
,
\end{tabular} & 0 & 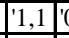 & $\begin{array}{l}0,0 \\
\end{array}$ & 0,0 & 0,0 & \\
\hline Realiza & \begin{tabular}{|c|c|c|}
0 & 10,0 \\
\end{tabular} & $' 0,0 \mid$ & 0,0 & 0,0 & 0,0 & \begin{tabular}{|l|l|} 
\\
\end{tabular} & \begin{tabular}{|l|l|} 
& \\
\end{tabular} & \begin{tabular}{|c|c|} 
& \\
\end{tabular} & \begin{tabular}{l|l}
0,0 \\
\end{tabular} & \begin{tabular}{l|l}
0,0 & 0 \\
0
\end{tabular} & '0,0 ' & \begin{tabular}{l|l}
0,0 & 1 \\
\end{tabular} & 0,0 & 0,0 & \begin{tabular}{|l|l|} 
& \\
\end{tabular} & \begin{tabular}{|c|c|} 
& \\
\end{tabular} & \begin{tabular}{l|l}
0,0 \\
\end{tabular} & \begin{tabular}{l|l}
0,0 & 0 \\
0
\end{tabular} & 0,0 & $|0,0|$ & $' 0,0 \mid$ & 0,0 & \begin{tabular}{|l|l|} 
\\
\end{tabular} & \begin{tabular}{l|l} 
\\
\end{tabular} & 0,0 & \begin{tabular}{l|l}
0,0 & 1
\end{tabular} & 0,0 & '2,0' & \begin{tabular}{|l|l|} 
\\
\end{tabular} & \begin{tabular}{|l|l|} 
& \\
\end{tabular} & 0,0 & '1, '1,0' & \begin{tabular}{|l|l|l}
0,0 & 1 \\
\end{tabular} & 1,0 & \begin{tabular}{|l|l}
1,0 & 0 \\
\end{tabular} & 0,0 \\
\hline & \begin{tabular}{c|c|c|}
$0,0,0$ \\
\end{tabular} & & $\mid 0,0$ & $\mid 0,0$ & 0,0 & \begin{tabular}{|l|l|} 
\\
\end{tabular} & \begin{tabular}{|l|l|} 
& \\
\end{tabular} & \begin{tabular}{|c|c|} 
& \\
\end{tabular} & \begin{tabular}{|c|c} 
\\
\end{tabular} & $\begin{array}{l}0,0 \\
0\end{array}$ & '0,0' & \begin{tabular}{l|l}
0,0 & 1 \\
\end{tabular} & 0,0 & 0,0 & \begin{tabular}{|l|l|} 
& \\
\end{tabular} & \begin{tabular}{|c|c|} 
& \\
\end{tabular} & \begin{tabular}{|c|c} 
\\
\end{tabular} & \begin{tabular}{l|l}
0,0 & \\
\end{tabular} & 0,0 & 10,0 & \begin{tabular}{|l|l|} 
\\
\end{tabular} & $\begin{array}{l} \\
\end{array}$ & \begin{tabular}{|l|l|} 
\\
\end{tabular} & \begin{tabular}{|c|c} 
\\
\end{tabular} & 0,0 & $\begin{array}{l}0,0 \\
0\end{array}$ & 0,0 & $10,0 \mid$ & \begin{tabular}{|l|l|} 
\\
\end{tabular} & \begin{tabular}{l|l}
1,0 \\
\end{tabular} & & '3,0' & \begin{tabular}{|l|l}
0,0 & 0 \\
\end{tabular} & 0,0 & 0,0 & $\overline{0,0}$ \\
\hline & \begin{tabular}{r|l}
0 & 10,0 \\
\end{tabular} & \begin{tabular}{|l|} 
\\
\end{tabular} & 10,0 & 0,0 & 0,0 & \begin{tabular}{|l|l|} 
\\
\end{tabular} & \begin{tabular}{|l|l|} 
& \\
\end{tabular} & \begin{tabular}{|c|c|} 
& \\
\end{tabular} & \begin{tabular}{l|l}
0,0 \\
\end{tabular} & \begin{tabular}{l|l}
0,0 & 0 \\
0
\end{tabular} & '0,0 'ו & \begin{tabular}{l|l}
0,0 & 0 \\
\end{tabular} & 0,0 & 0,0 & \begin{tabular}{|l|l|} 
& \\
\end{tabular} & \begin{tabular}{|c|c|} 
& \\
\end{tabular} & \begin{tabular}{l|l}
0,0 \\
\end{tabular} & \begin{tabular}{l|l}
0,0 & 0 \\
\end{tabular} & 0,0 & $|0,0|$ & \begin{tabular}{|l|l|} 
\\
\end{tabular} & 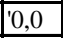 & \begin{tabular}{|l|l|} 
\\
\end{tabular} & \begin{tabular}{l|l} 
\\
\end{tabular} & '0,0' & \begin{tabular}{l|l}
0,0 & 1
\end{tabular} & 0,0 & 10,0 & \begin{tabular}{|l|l|} 
\\
\end{tabular} & \begin{tabular}{l|l}
0,0 & 1 \\
\end{tabular} & 0 & ' & \begin{tabular}{|l|l}
0,0 & 1 \\
\end{tabular} & $1,1,0$ & & \\
\hline ltruí & \begin{tabular}{|c|c|c|}
0 & 1 \\
\end{tabular} & \begin{tabular}{|c|} 
\\
\end{tabular} & 0,0 & 0,0 & 0,0 & \begin{tabular}{|l|l|} 
\\
\end{tabular} & \begin{tabular}{|l|l|} 
& \\
\end{tabular} & \begin{tabular}{|c|c|} 
& \\
\end{tabular} & \begin{tabular}{|l|l|} 
\\
\end{tabular} & \begin{tabular}{l|l}
0,0 & 0 \\
\end{tabular} & '0,0 & \begin{tabular}{l|l}
0,0 \\
\end{tabular} & 0,0 & 0,0 & \begin{tabular}{|l|l|} 
& \\
\end{tabular} & \begin{tabular}{|l|l|} 
& \\
\end{tabular} & \begin{tabular}{|l|l|} 
\\
\end{tabular} & \begin{tabular}{l|l}
0,0 \\
\end{tabular} & 0,0 & $' 0,0$ & '0,0 & '0,0 & \begin{tabular}{|l|l|} 
\\
\end{tabular} & $\begin{array}{l} \\
\end{array}$ & 0,0 & \begin{tabular}{l|l}
0,0 & 0 \\
\end{tabular} & 0,0 & '0,0' & \begin{tabular}{|l|l|} 
& \\
\end{tabular} & \begin{tabular}{|l|l|} 
& \\
\end{tabular} & 0,0 & \begin{tabular}{|c|c|c|} 
& \\
\end{tabular} & \begin{tabular}{|l|l|l} 
& 0,0 \\
\end{tabular} & $\begin{array}{l}0,0 \\
\end{array}$ & \begin{tabular}{|l|l|l|}
0,0 & 0 \\
\end{tabular} & 0,0 \\
\hline & \begin{tabular}{|c|c|c|}
0 & 10,0 \\
\end{tabular} & '0,0 & 0,0 & 0,0 & 0,0 & '0,0|' & \begin{tabular}{|c|} 
\\
\end{tabular} & \begin{tabular}{|c|c|} 
& \\
\end{tabular} & \begin{tabular}{|l|l} 
\\
\end{tabular} & $\begin{array}{l}0,0 \\
0\end{array}$ & '0,0' & \begin{tabular}{l|l}
0,0 & 1 \\
\end{tabular} & 0,0 & 0,0 & \begin{tabular}{|c|} 
\\
\end{tabular} & \begin{tabular}{|c|c|} 
\\
\end{tabular} & & $\begin{array}{l}0,0 \\
0\end{array}$ & 0 & $' 0,0 \mid$ & & \begin{tabular}{|l|} 
\\
\end{tabular} & '0,0|' & & '0,0' & $\begin{array}{l}0,0 \\
0\end{array}$ & 0,0 & \begin{tabular}{|c|} 
\\
\end{tabular} & & & & , 0,0 & \begin{tabular}{l|l}
0,0 & 0 \\
\end{tabular} & 0 & & $\overline{0,0}$ \\
\hline atid & \begin{tabular}{l|l|l}
0 & 0,0 \\
\end{tabular} & ' 0,0 ' & $\mid 0,0$ & \begin{tabular}{|l|}
0,0 \\
\end{tabular} & 0,0 & '0,0|' & \begin{tabular}{|c|c|} 
\\
\end{tabular} & \begin{tabular}{|l|l|} 
\\
\end{tabular} & \begin{tabular}{l|l|l} 
\\
\end{tabular} & \begin{tabular}{l|l|l}
0,0 & \\
\end{tabular} & ' & \begin{tabular}{l|l}
0,0 & 0 \\
\end{tabular} & 0,0 & 0,0 & \begin{tabular}{|l|l|} 
& \\
\end{tabular} & \begin{tabular}{|l|l|} 
& \\
\end{tabular} & \begin{tabular}{|l|c|} 
\\
\end{tabular} & \begin{tabular}{l|l}
0,0 \\
\end{tabular} & 0,0 & $|0,0|$ & $10,\left.0\right|^{\prime}$ & 0,0 & $|0,0|$ & \begin{tabular}{|l|c|} 
\\
\end{tabular} & 0,0 & \begin{tabular}{l|l}
0,0 & 1 \\
0
\end{tabular} & 0,0 & $\mid 0,0$ & \begin{tabular}{|l|l|} 
\\
\end{tabular} & \begin{tabular}{|l|l|} 
& \\
\end{tabular} & 0,0 & 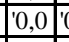 & \begin{tabular}{l|l}
0,0 & 0 \\
\end{tabular} & 0 & & \\
\hline espeito & \begin{tabular}{|c|c|c|}
0 & 1 \\
\end{tabular} & $\left|{ }^{\prime} 0,0\right|$ & $|0,0|$ & $|0,0|$ & 0,0 & $|0,0|$ & '| & \begin{tabular}{|l|l|} 
& \\
\end{tabular} & $\begin{array}{l} \\
\end{array}$ & $\begin{array}{l} \\
\end{array}$ & ' & \begin{tabular}{l|l}
0,0 & 0 \\
\end{tabular} & \begin{tabular}{|l|l|}
0,0 \\
\end{tabular} & 0,0 & \begin{tabular}{|l|l|} 
& \\
\end{tabular} & \begin{tabular}{l|l|} 
\\
\end{tabular} & $\begin{array}{l} \\
\end{array}$ & $\begin{array}{l} \\
\end{array}$ & 0,0 & $|0,0|$ & $|0,0|$ & \begin{tabular}{|l|l} 
\\
\end{tabular} & & $\begin{array}{l} \\
\end{array}$ & '0,0' & $\begin{array}{l}0,0 \\
\end{array}$ & 0,0 & $|0,0|$ & ' & '|' & 0,0 & 0 & \begin{tabular}{l|l}
0,0 & 0 \\
\end{tabular} & 0,0 & & \\
\hline
\end{tabular}

\begin{tabular}{|l|}
\hline Gratidão \\
\hline Respeito \\
\hline
\end{tabular}

\section{Revista Administração em Diálogo}

ISSN 2178-0080

Programa de Estudos Pós-Graduados em Administração Pontifícia Universidade Católica de São Paulo 
Tayanne Ferraz da Silva Poberschnigg, Márcio Lopes Pimenta. RAD Vol.17, n.3, Set/Out/Nov/Dez 2015, p.59-86.

\author{
Fonte: Dados da pesquisa
}

Revista Administração em Diálogo

ISSN 2178-0080

Programa de Estudos Pós-Graduados em Administração

Pontifícia Universidade Católica de São Paulo 


\section{Mapa Hierárquico de Valor}

A fim de que essas conexões fossem melhor visualizadas, elaborou-se um mapa hierárquico de valor, representado pela Figura 2. Considerou-se o ponto de corte 3 na relação entre os elementos, tendo um aproveitamento de $75 \%$ das ligações totais, como recomenda Reynolds e Gutman (I988). Assim, alguns elementos apresentados na matriz de implicação não estão presentes no mapa, pois não atingiram pelo menos 3 ligações e foram descartados.

Figura 2 - Mapa Hierárquico de Valor

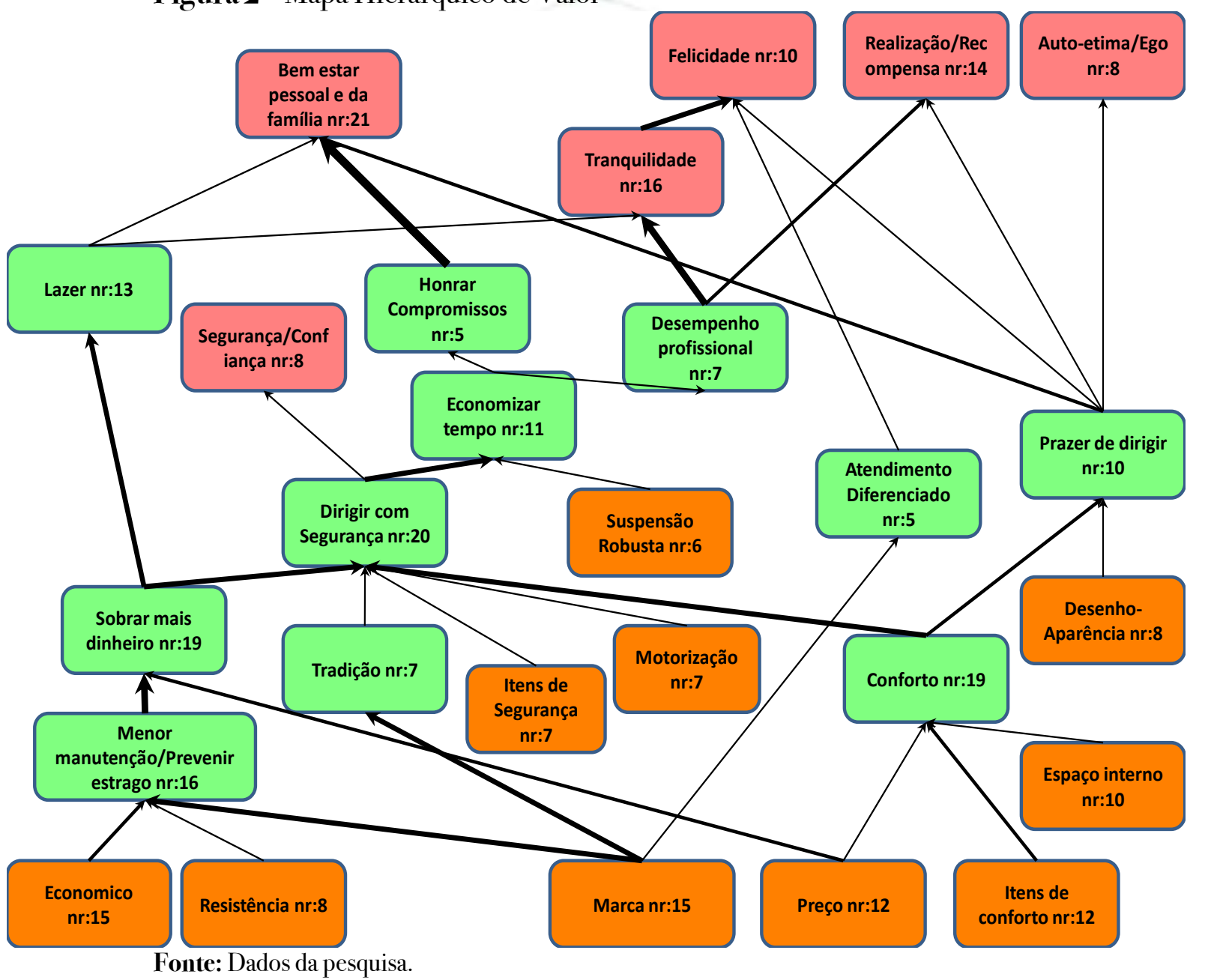

Por meio deste mapa cognitivo, é possível identificar as principais cadeias adquiridas com o propósito de compreender, por meio da interpretação das conexões, o comportamento do público pesquisado em relação à compra de veículos. Os atributos estão representados pelo retângulo de cor laranja, as consequências pelo verde e os

\section{Revista Administração em Diálogo} ISSN 2178-0080

Programa de Estudos Pós-Graduados em Administração Pontifícia Universidade Católica de São Paulo 
valores pelo rosa. No mapa constam: a codificação de cada elemento, em números, e também, o número de incidências (nr). As cadeias dominantes estão representadas pelos traços de ligação mais espessos que, conforme Reynolds e Gutman (I988), podem explicar os comportamentos predominantes que levam os clientes a escolherem determinado automóvel ou marca na região pesquisada.

Foram identificadas 68 cadeias, dentre as quais merecem destaque as oriundas do atributo 6, "Econômico", com 6 cadeias; 8, "Itens de Conforto", com 8 cadeias; Io, "Marca", com II cadeias; e ı2, "Preço", com I4 cadeias. Estes atributos geram as seguintes cadeias dominantes: 6-23-26-2I-3I, 6-23-26-I8-I9-2O-3I, 6-23-26-I8-I9-I7-37-

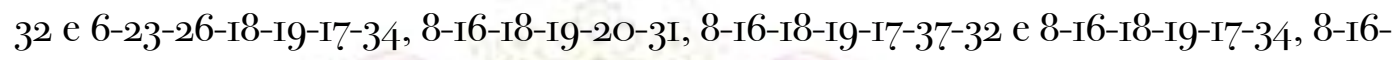
25-3I, IO-23-26-2I-3I, IO-23-26-I8-I9-2O-3I, IO-23-26-I8-I9-I7-37-32 е IO-23-26-I8-I9I7-34, ІО-28-I8-I9-2О-3І, ІО-28-І8-I9-I7-37-32 е ІО-28-І8-I9-І7-34, І2-26-2I-3І, І2-26-

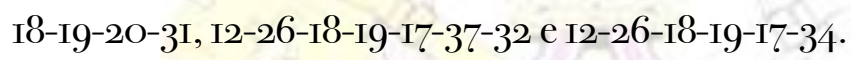

Percebe-se que os atributos 6,8 , IO e I2 têm em comum as consequências finais $2 \mathrm{O}$ e I7, ambas ligadas a I9, as quais resultam nos valores 3 I, 37, 32 e 34 . Isso significa que essas cadeias, dado o elevado número de incidências, determinam o comportamento de compra dos clientes entrevistados. A análise da cadeia I2-26-2I-3I, por exemplo, permite dizer que o consumidor deseja comprar um carro com um preço mais acessível e compatível com sua renda (I2) a fim de economizar dinheiro ao evitar um gasto maior na compra (26) para que possa investir em viagens, gastos com moradia, roupa, alimentação, entre outros (2I), gerando uma sensação de bem-estar para o cliente que preza a qualidade de vida (3I).

As cadeias 6-23-26-I8-I9-I7-37-32 e 6-23-26-I8-I9-I7-34 podem ser interpretadas da seguinte forma: comprar um automóvel econômico (6) pode relacionarse a comprar um carro que não dê muitas despesas com manutenção ou que tenha peças mais baratas para reposição (23). Dessa forma, evitam gastos para que sobre mais dinheiro (26) e não se tenha tantas preocupações financeiras, permitindo que o condutor dirija com mais segurança, devido à redução do estresse, e tenha mais confiança nas ultrapassagens (I8); essas ultrapassagens resultam em um ganho de tempo para o indivíduo (I9) e proporcionam que o mesmo atenda mais clientes (I7). Assim, a pessoa se sente aliviada por conseguir melhorar o desempenho profissional e possivelmente a sua 
renda (37) o que contribui para a sua própria felicidade (32), pois não precisa ficar preocupada com questões financeiras e/ou com mau desempenho; ou então, se sente realizada por conseguir atender mais clientes, proporcionando uma sensação de dever cumprido (34).

Já a cadeia 8-I6-25-3I explica que carros que possuem direção hidráulica, vidro elétrico, ar condicionado e bancos macios (8) são importantes porque proporcionam certo conforto ao consumidor (I6), permitindo que ele tenha prazer ao conduzir o veículo (25) e se sinta bem (3I). Por fim, como exemplo de cadeia originada pelo atributo IO, tem-se a IO-28-I8-I9-2O-3I a qual indica que o consumidor procura comprar um automóvel de marca confiável (IO), seja por ter tradição no mercado ou por ser recomendada por parentes e conhecidos (28). Dessa forma, o condutor percebe estabilidade ao dirigir e confiança no carro para realizar viagens e ultrapassagens (I8), as quais acarretam em uma economia de tempo (I9) e possibilitam que a pessoa chegue aos locais no horário combinado (20), gerando o bem-estar de honrar um compromisso (3I).

Portanto, os indivíduos procuram carros econômicos, com preços atrativos, de marca conhecida ou tradicional e que têm itens de conforto porque acreditam que, por meio desses atributos, conseguirão sentir prazer ao dirigir, ter mais dinheiro para gastar com lazer, mais tempo para não chegarem atrasados ou para se desenvolverem profissionalmente. Por meio destas consequências, os consumidores acreditam que poderão alcançar o bem-estar pessoal e familiar, a realização pessoal e a felicidade, sendo esta relacionada ao sentimento de tranquilidade. O quadro a seguir apresenta o resumo das conexões entre os atributos e valores encontrados nessa pesquisa. 
Quadro3- Resumo da conexão entre atributos e valores

Atributos

Ligações com consequências e valores

\begin{tabular}{|c|c|}
\hline Suspensão Robusta & $\begin{array}{l}\text { Um carro com suspensão robusta economiza tempo devido à facilidade de dirigir em } \\
\text { estradas de terra. Isso faz com que os clientes cumpram seus compromissos e se sintam } \\
\text { bem, ao mesmo tempo em que podem aplicar o seu tempo para se desenvolverem } \\
\text { profissionalmente, sentindo-se tranquilos, felizes e realizados. }\end{array}$ \\
\hline Desenho/Aparência & $\begin{array}{l}\text { As pessoas escolhem um carro que tem determinado design ou cor porque isso faz com } \\
\text { que se sintam bem e felizes ao dirigir, estando ligado, também, à realização de ter um } \\
\text { carro que se almeja e ao aumento da (o) autoestima/ego. }\end{array}$ \\
\hline Econômico & $\begin{array}{l}\text { Um carro econômico evita que os consumidores tenham gastos. Essa economia pode ser } \\
\text { aplicada no lazer ou ser utilizada para pagar contas, reduzindo o estresse e possibilitando } \\
\text { que o indivíduo trabalhe mais, o que gera sentimentos de bem-estar, segurança, } \\
\text { realização, tranquilidade e felicidade. }\end{array}$ \\
\hline Espaço Interno & $\begin{array}{l}\text { O consumidor almeja carros com um espaço interno adequado para se sentir confortável, } \\
\text { a fim de ter prazer de dirigir ou evitar o cansaço, estando mais disposto para o trabalho. } \\
\text { Assim, busca alcançar o bem-estar, a segurança, a realização pessoal, a (o) } \\
\text { autoestima/ego, a tranquilidade e a felicidade. }\end{array}$ \\
\hline Itens de Conforto & $\begin{array}{l}\text { Carros que possuem direção hidráulica e bancos macios, por exemplo, deixam o cliente } \\
\text { mais confortável, reduzindo esforços nas manobras e proporcionando uma confiança } \\
\text { maior na direção. Os valores, portanto, envolvem o bem-estar, a segurança, a realização } \\
\text { pessoal, a (o) autoestima/ego, a tranquilidade e a felicidade. }\end{array}$ \\
\hline Itens de Segurança & $\begin{array}{l}\text { Itens de segurança evitam que o motorista se sinta inseguro, tendo mais confiança ao } \\
\text { dirigir. Dessa forma, o indivíduo busca um carro que lhe faça sentir bem, seguro, } \\
\text { realizado, tranquilo e feliz. }\end{array}$ \\
\hline Marca & $\begin{array}{l}\text { Os entrevistados acreditam que ao comprarem um carro de marca conhecida, não terão } \\
\text { tantos problemas com atendimento nem com a qualidade. São pessoas que valorizam o } \\
\text { bem-estar, a segurança, a realização pessoal, a tranquilidade e a felicidade. }\end{array}$ \\
\hline Motorização & $\begin{array}{l}\text { Os benefícios esperados ao ter um automóvel potente, com boa capacidade de } \\
\text { aceleração, são: segurança, bem-estar, realização, tranquilidade e felicidade. }\end{array}$ \\
\hline Preço & $\begin{array}{l}\text { Clientes que prezam o custo-benefício e visam obter economia financeira e conveniência, } \\
\text { valorizam o bem-estar, a segurança, a realização pessoal, a (o) autoestima/ego, a } \\
\text { tranquilidade e a felicidade. }\end{array}$ \\
\hline Resistência & $\begin{array}{l}\text { Um automóvel robusto é mais durável, evitando gastos com manutenção e permitindo } \\
\text { que o consumidor invista tempo e dinheiro em outras atividades. Valoriza-se a segurança, } \\
\text { o bem-estar, a realização, a tranquilidade e a felicidade. }\end{array}$ \\
\hline
\end{tabular}

Fonte: Dados da pesquisa.

Relacionando-se este estudo com outros apresentados na revisão de literatura, percebe-se que alguns atributos identificados nesta pesquisa, como "Marca", "Preço", "Econômico", "Desenho/Aparência" e "Itens de Segurança", são semelhantes com aqueles encontrados em estudos dos autores Mueller e Haan (2009), Fernandes (2007), Araújo, Araújo e Alexandre (2OII), Campos e Ramos (2002) e Lemos e Slongo (2OII). Já

\section{Revista Administração em Diálogo} ISSN 2178-0080 
para os valores, tem-se que os resultados desta pesquisa são compatíveis com as realizadas pelos autores Porto e Torres (2014), Fernandes (2007), Escudero e Prado (2008) e Nunes, et. al. (2OI2), no que se refere aos valores "Autoestima/Ego", "Bemestar pessoal e da família", "Felicidade", "Realização" e "Segurança/Confiança".

Como diferencial, baseando-se nos quatro domínios de valor propostos por Schwartz (1992), é perceptível que a maioria dos consumidores de automóveis que responderam a essa pesquisa tende a ter valores individuais relacionados à dimensão "autoaprimoramento", pois busca o bem-estar pessoal e/ou familiar, aproveitar a vida, sentir prazer e ter sucesso.

Além disso, ao observar-se o mapa hierárquico de valor, é perceptível que a "marca" é o atributo mais relevante para os clientes, como salientado pelos autores Fantini (2009) e Gonçalves Filho, Souki e Gonçalves (2009) em seus estudos, o que comprova a afirmação feita por Oliveira, et. al., (20IO), de que a mesma é o principal vínculo entre uma entidade e o público-alvo.

O processo de decisão de compra destes consumidores pode ser considerado complexo, como alertam Mueller e Haan (2009), pois foram encontrados diversos fatores que interferem no comportamento de compra, estando relacionados ao desempenho e à resistência do carro, ao design, ao conforto e ao preço. A partir dos dados desta pesquisa, é possível dizer que a escolha de um automóvel depende da intensidade com que as pessoas se identificam com ele e sua marca e, também, se as mesmas acreditam que o bem lhes trará os benefícios almejados. Essa afirmação condiz com as ideias de Ramalho e Ayrosa (2009), que destacam a crescente necessidade do estudo da conexão entre veículos automotores e valores pessoais.

\section{Conclusões}

A compreensão das necessidades dos clientes é fundamental para organizações que almejam ser competitivas no mercado. O estudo do comportamento do consumidor tem, portanto, a função de auxiliar as entidades no aprimoramento ou desenvolvimento de bens, de forma que o produto final corresponda às expectativas do público-alvo. Além disso, possibilita identificar os fatores cruciais na decisão de compra e, então, ações

\section{Revista Administração em Diálogo} ISSN 2178-0080 
podem ser movidas na segmentação do consumidor, no atendimento ao cliente e na elaboração de propagandas estratégicas.

Essa pesquisa teve por base o mercado consumidor de automóveis de Uberlândia, Triângulo Mineiro, procurando saber como se caracteriza a relação entre os atributos de veículos automotores com os valores pessoais, e como a explicação dessa relação ajuda a entender o comportamento deste tipo de consumidor. Foram identificados dez atributos no mapa hierárquico de valor, dos quais se destacaram os quatro primeiros: "Econômico" (6), "Marca" (Іо), "Preço" (г2), "Itens de Conforto" (8), "Suspensão Robusta" (4), "Desenho/Aparência" (5), "Espaço Interno" (7), "Itens de Segurança” (9), "Motorização" (II) e "Resistência" (І3). Os consumidores acreditam que os automóveis que possuem esses atributos lhes proporcionam os valores "Bem-estar", "Realização", "Felicidade" e "Tranquilidade", com exceção do atributo "Desenho/Aparência" que não gera o sentimento de "Tranquilidade".

Os entrevistados acreditam indivíduos alcançar o valor "Segurança" por meio de todos os atributos que formam o mapa hierárquico de valor, salvo "Suspensão Robusta" e "Desenho/Aparência", pois as características dos carros fazem com que as pessoas possam "Evitar Transtornos". O valor "Autoestima/Ego" é atingido por meio dos atributos 5, 7, 8 e I2, os quais proporcionam "Prazer de Dirigir”. Dessa forma, valores pessoais são itens determinantes no processo de decisão de compra de veículos automotores, conforme a opinião dos clientes que participaram da pesquisa.

\section{Principais Interpretações Teóricas}

Como podem ser observadas nas cadeias dominantes (REYNOLDS; GUTMAN, I988), as conexões entre os elementos levaram a entender que os entrevistados procuraram carros econômicos, de uma marca conhecida e com preço atraente porque buscam economia financeira. Dessa forma, podem investirem seu dinheiro para o próprio bem-estar ou da família e/ou para realizar atividades que lhes proporcionem tranquilidade (valor instrumental), colaborando, assim, para a sua felicidade (valor terminal), conforme o arcabouço teórico de Rokeach (I973).

Por outro lado, os respondentes podem querer economizar para não se sentirem estressados por razões financeiras e, com isso, ficando despreocupados e confiantes para

\section{Revista Administração em Diálogo} ISSN 2178-0080 
dirigir. Ao evitar esse transtorno, estes se sentem seguros para realizar ultrapassagens, o que gera um sentimento de bem-estar. Acreditam, também, que essa consequência permite dirigir de forma veloz e conseguir chegar a tempo aos compromissos, gerando felicidade e realização por poderem trabalhar mais e melhor.

Esses consumidores também buscaram carros que lhes oferecessem itens de conforto, com a finalidade de sentirem bem-estar devido ao prazer de dirigir ou de não se sentirem incomodados na direção. Esse fato também proporciona estarem seguros ao realizarem ultrapassagens as quais lhes poupam tempo para melhorar o desempenho no trabalho. Enfim, os entrevistados possuem, principalmente, valores individuais ligados a poder, realização e hedonismo, segundo a escala de Schwartz (I992), pois anseiam ter sucesso profissional, bem-estar e felicidade.

\section{Interpretações do Ponto de Vista Gerencial}

Os aspectos apresentados ressaltam a importância de conhecer a estrutura cognitiva dos consumidores a fim de compreender seu comportamento de compra, como também amplia as bases para a elaboração da estratégia de marketing das concessionárias, utilizando-se de informações mais eficazes do que variáveis demográficas, por exemplo. A estrutura de valores dos consumidores pode, portanto, ajudar: I) na definição de bases para segmentação de mercado, caracterizando grupos de consumo; 2) no desenvolvimento de novos produtos, a partir da definição de atributos relevantes; e 3) na comunicação com o cliente, através da compreensão aprofundada de sua percepção e de suas necessidades.

\section{Limitações e Sugestões para Pesquisas Futuras}

É importante destacar que a pesquisa teve limitações quanto à abrangência do estudo, que se baseou nas percepções dos consumidores de uma concessionária em Uberlândia, não podendo seus resultados ser generalizados. Outra limitação envolve a ausência de critérios para a escolha dos sujeitos de pesquisa, exceto serem clientes da concessionária em questão. Apesar da caracterização permitida pela aplicação do questionário sócio-demográfico, não há conclusões específicas de consumo como em investigações realizadas com somente pessoas de sexo feminino ou masculino e 
consumidores de determinado tipo de carro. Contudo, os resultados desta pesquisa são contribuições consistentes e relevantes para o estudo do comportamento do consumidor de automóveis, pois apontam o que os clientes desejam ao adquirirem um automóvel e condizem com os argumentos de outros autores pesquisados.

Como sugestão para futuras pesquisas, seria interessante que fossem feitas entrevistas com consumidores de outras concessionárias para comparar resultados e verificar se há diferenças significativas entre o comportamento do consumidor de veículos automotores neste sentido. Outra recomendação para futuras pesquisas referese ao estudo de fidelidade à marca, avaliando-se o que leva o consumidor a escolher determina marca em detrimento à outra e ser fiel a mesma. Ainda, sugere-se que novos estudos sejam realizados de modo mais aprofundado, estabelecendo critérios mais específicos para a escolha dos sujeitos de pesquisa, como sexo, idade, renda, região, escolaridade e modelo de carro adquirido.

\section{Referèncias}

ANDRADE, Maria Margarida de. Introdução à metodologia do trabalho científico: elaboração de trabalhos na graduação. 4 ed. São Paulo: Atlas, 1999.

ANFAVEA. Anuário da Indústria Automobilística Brasileira. ANFAVEA, São Paulo, 2OI4.

Disponível em: <http://www.anfavea.com.br/anuario.html>. Acesso em: I5 abr. 20I5.

ARAÚJO, Richard Medeiros; ARAÚJO, Adriel Medeiros; ALEXANDRE, Mauro Lemuel. Decisão de Compra de Veículos Novos por Estudantes Universitários: Fatores e Influência. Qualit@s Revista Eletrônica, v. I2, n. 2, 201 I.

BAKER, S.; THOMPSON, K. E.; ENGELKEN, J. Mapping the values driving organic food choice: Germany vs the UK. European Journal of Marketing: v. $3^{8}$ n. 8, p. 995-IOI2, 2004.

BAUER, Martin W.; GASKELL, George. Pesquisa qualitativa com texto: imagem e som: um manual prático. Tradução de Pedrinho A. Guareschi. 2 ed. Rio de Janeiro: Vozes, 2003.

BLACKWELL, R.D.; MINIARD, P.W.; ENGEL, J.F. Comportamento do consumidor. 9. ed. São Paulo: Thompson Learning, 2005. 6o6p.

CAMPOS, Antonio Braulio Figueiredo; RAMOS, Rubens Eugênio Barreto. Fatores que Afetam a Satisfação do Cliente: Estudo de Caso com Compradores de Automóveis. XXII Encontro Nacional de Engenharia de Produção, Curitiba, outubro 2002.

CARDOSO, Marcos Antonio; KISTMANN, Virginia Borges. Modularização e Designe na Indústria Automotiva: O Caso do Modelo Fox da Volkswagen do Brasil. Revista Produção Online, v. 8, n. 4, 2008. 
CARVALHEIRO, João Tiago da Cruz. Antecedentes do comprometimento afetivo com automóveis de luxo. Dissertação apresentada como requisito necessário para obtenção do título de Mestre em Gestão do Departamento de Economia, Gestão e Engenharia Industrial da Universidade de Aveiro, $2 \mathrm{OI2}$.

CERQUEIRA, Lucas Santos; SILVA, Afonso Bahiense da; FARIAS, Vitor Tavares. Consumidor de Baixa Renda: Uma Analise dos Fatores que Influenciam a Aquisiçao de Automoveis em Salvador. Revista de Administração e Contabilidade da FAT, v. 5, n. 2, p. III-I28, 2 OI3.

CHAO, P.; GUPTA, P. B. Information search and efficiency of consumer choices of new cars Country-of-origin effects. International Marketing Review, I2 (6), 47-59, 1995.

DIAS, M. B.; OLIVEIRA-CASTRO, J. M. Comportamento de procura por produtos: efeitos da quantidade de marcas. Revista Psicologia, Organização do Trabalho. v.6, n.I, Florianópolis jun. 2006.

ESCUDERO, Fabiana Thiele; PRADO, Paulo Henrique Muller. Analise das Metas do Consumidor: Uma Contribuição Metodológica. RAE-eletrônica, v. 7, n. 2, jul/dez 2008.

FANTINI, M. J. Antecedentes e intenções comportamentais do amor a marca de consumidores: um estudo empírico no mercado automotivo. Dissertação apresentada ao Curso de Mestrado em Administração, da Faculdade de Ciências Empresariais da Universidade Fumec, 2009.

FERNANDES, B. P. L. Segmentação de consumidores no Brasil: um estudo empírico no mercado automotivo. Dissertação apresentada como requisito parcial para obtenção do título de Mestre em Administração do Curso de Mestrado em Administração da Universidade FUME, 2007.

FOXALL, G. R.; OLIVEIRA-CASTRO, J. M; JAMES, V.K.; SCHREZENMAIER, T.C. Consumer behavior analysis: the case of brand choice. Revista Psicologia Organizações e Trabalhos, v. 6, p. $5^{2-}$ 8o, 2006.

GIL, A. C. Métodos e Técnicas de Pesquisa Social. 5 ed. Atlas. São Paulo, I999.

GIL, A. C. Como elaborar projetos de pesquisa. 4. ed. São Paulo: Atlas, 2002.

GONÇALVES FILHO, Cid; SOUKI, Gustavo Quiroga; GONÇALVES, Carlos Alberto. Valor das Marcas (Brand Equity) para Consumidor: desenvolvimento e validação de um instrumento de mensuração no setor automotivo. RECADM - Revista Eletrônica de Ciência Administrativa, v.8, n. I, p. IO6-II8, maio 2009.

GROHMANN, Márcia Zampieri; BATTISTELLA, Luciana Flores; SCHOEDLER, Andréia Regina. Atributos importantes para o consumidor de automóveis: classificação em função instrumental ou expressiva. Revista de Administração FACES Journal, v. II, n. I, $2 \mathrm{OI} 2$.

GUTMAN, J. A means end chain model based on consumer categorization processes. Journal of Marketing, v .46, n. 2, p.6o-72. Spring I982.

GUTMAN, J. Exploring the nature of linkages between consequences and values. Journal of Business Research, New York, v. 22 n. 2, p.I43-I48. I99I.

LEMOS, Ricardo Felipe; SLONGO, Luiz Antonio. Atributos de Compra de Automóveis de Alto Valor: Uma Abordagem Comparativa Sob a Ótica do Vendedor e do Comprador. MCTRES.

Disponível em

$<$ http://www.mctres.com.br/mc3/conteudo/Avaliacaodeatributosdeveiculosdealtovalorcomparand oaotica.doc $>$. Acesso em I5 de julho de 20 II. 
MOYANO, Carlos Mello; LENGLER, Jorge. Explorando as relações entre os valores pessoais, autoconceito dimensão afetiva de segurança e comportamento feminino de consumo: perspectivas a partir da experiência de varejo brasileiro. Revista de Administração FACES Journal, v. ı2, n. I, p. II8I37, 2013 .

MUELLER, M. G.; HAAN, P. How much do incentives affect car purchase? Agent-based microsimulation of consumer choice of new cars - Part I: Model structure, simulation of bounded rationality, and model validation. Energy Policy, v.37, 2009, pp.IO72-IO82.

MURALIE, Sushma; MITTAL, Sanjiv. Values and their Influence on Attitudinal Segments Identified amongst Automobile Users. Asia Pacific Business Review, v. 7, n. 2, p. I47-I59, 2 OII.

NASPETTI, S.;ZANOLI, R. Do Consumers Care About Where They buy Organic Products? A Means-End Study with Evidence from Italian Data. In: Baourakis, G. (ed.). Marketing Trends for Organic Food in the 2IstCentury. I. ed. Cingapura: World Scientific, 2004, v. 3, p.238-255.

NUNES, N. S.; PINTO, L. A.; MURAKAMI, L. C.; PIMENTA, M. L. Valores associados à compra de automóvel por jovens no Ceará: um estudo sob a ótica da cadeia de meios-fim. Revista Brasileira de Marketing, v. II, n. 3, p. IOI-I23, $2 \mathrm{OI} 2$.

OLIVEIRA, M. E. R.; GALLI, L. C. L. A.; CÔNSOLI, M. A.; CASTRO, L. T. e. Gestão de marcas emocionais: um estudo exploratório no setor automotivo de veículos leves. REMark-Revista Brasileira de Marketing, São Paulo, v. 9, n. I, p. 86-ıо4, jan./abr. 20 öo.

PIMENTA, Márcio L.; MELO, Daniela de Castro; VILAS BOAS, Luis H. de Barros; SILVA, Andrea L. da; SETTE, Ricardo de Souza. Bases de segmentação por valores: um estudo sobre o mercado consumidor de orgânicos da cidade de Uberlândia. Revista Teoria e Evidência Econômica, ano I4, n. 3I, p. I42-I65, jul./dez. 2008.

PIMENTA, M.L.; PIATO, E.L.; VILAS BOAS, L.H.B. MORIGUCHI, S.N. Sabor e bem-estar: uma relação entre atributos de produto e valores pessoais de consumidores de marcas regionais de café. Brazilian Business Review, v.9, n.3, p.I22-I44, 2012.

PORTO, Rafael Barreiros. Valores Humanos Pessoais e Significados do Produto como Preditores de Preferência por Tipos de Automóveis. Dissertação de mestrado apresentado ao Instituto de Psicologia da Universidade de Brasília, como requisito parcial à obtenção do título de mestre em Psicologia, 2005.

PORTO, Rafael Barreiros; TORRES, Cláudio V. Valores humanos como moderadores e supressores na preferência do consumidor por marcas e produtos. Estud. psicol.(Natal), v. I9, n. 2, p. 9I-IOI, 2 OI4.

RAMALHO, R.; AYROSA, E. A. T. Subcultura Tuning: a identidade estendida na personalização de automóveis. Revista de Ciências da Administração, v. II, n. 24, p. I69-I94, maio/ago 2009.

REYOLDS, Thomas J.; GUTMAN, Jonathan. Laddering Theory, Method, Analysis, and Interpretation. Journal of Advertising Research. p. II-3I. February/March, I988.

ROKEACH, M. The nature of human values. New York: Free Press, I973.

SAMPAIO, Danilo de Oliveira; VISCARDI, Adriana W.; ORNELAS, Rubens; NASCIMENTO, Adilson Felismino; CASTRO, Cláudio V. L.; AFONSO, Daniel F.; BRAVO, Emílio C. de Freitas; SILVA, Roberto Ferreira Tavares. Um Estudo Comparativo Sobre o Comportamento do Consumidor de Automóveis Novos. Anais do VII SEMEAD, Marketing, agosto 2004. 
SCHWARTZ, S. H. Universals in the content and structure of values: theory and empirical tests in 20 countries. Advances in Experimental Social Psychology, New York, v. 25, p. I-65, 1992.

SOARES, E. Metodologia Científica: lógica, epistemologia e normas. São Paulo: Atlas, 2003.

VELUDO-DE-OLIVEIRA, Tania M.; IKEDA, Ana Akemi. Usos e Limitações do Método Laddering. Revista de Administração Mackenzie. Ano 5, n. I. p. 197-222, 2004.

VELUDO-DE-OLIVEIRA, Tânia M.; IKEDA, Ana Akemi. O conceito de valor para o cliente: definições e implicações gerenciais em marketing. GESTÃO. Org-Revista Eletrônica de Gestão Organizacional, v. 3, n. I, 2 OIO.

VINSON, Donald E.; SCOTT, Jerome E.; LAMONT, Lawrence M. The Role of Personal Values in Marketing and Consumer Behavior. Journal of Marketing, abril I977. 